\title{
18. STABLE ISOTOPE RECORD OF HOLE 504 SEDIMENTS: HIGH RESOLUTION RECORD OF THE PLEISTOCENE 1
}

\author{
N. J. Shackleton and M. A. Hall, The Godwin Laboratory, University of Cambridge, Free School Lane, \\ Cambridge CB2 3RS, United Kingdom
}

\begin{abstract}
A detailed oxygen isotope record (resolution: about 2500 years) has been obtained for the Pleistocene sediments at Hole 504. Preliminary measurements made deeper in the section suggest that at least the upper Pliocene section is also amenable to detailed stable isotope work.

The record for the middle Pleistocene resembles that obtained previously from piston cores in the western equatorial Pacific, although the superior resolution of this high-accumulation-rate site reveals a greater amplitude of isotope variation than previously observed. The record for the lower Pleistocene reveals variation that is both greater in amplitude and higher in frequency than apparent from previously analyzed piston cores. The site provides the best material recovered to date for the study of the evolution of climatic variability during the past few million years.
\end{abstract}

\section{INTRODUCTION}

Hole 504 was in the Panama Basin on crust 5.9 m.y. old (a date based on magnetic anomalies). The chief objective of the drilling at the site, which was performed with the hydraulic piston corer, was to obtain information about the crust, but the sediment was also recovered and analyzed. We present here the results of (1) analyses of the upper 16 cores, which were sampled at 10 -cm intervals, and (2) a preliminary examination of the lower part of the section, which was sampled about every 1.5 meters. Isotope analyses have been published for a number of conventional piston cores from the Panama Basin region (Ninkovich and Shackleton, 1975; and Shackleton, 1977b), a region of high surface productivity and hence a higher-than-average rate of sediment accumulation. The high rate of sediment accumulation is advantageous for purposes of study because in many regions of low accumulation rate bioturbation obscures important features of the climatic record. The disadvantage of the region is that for a conventional piston corer the depth of the sediment precludes the penetration of lower Pleistocene sediments; thus, the drilling at Hole 504 provided a highly unusual opportunity to examine a high resolution record of the lower Pleistocene and upper Pliocene.

\section{METHODS}

The analytical methods we used have become more or less standard in stable isotope analysis. Samples were disaggregated by shaking them on a commercial orbital shaker in distilled water and washing them through a 150-micron sieve. Although both the coarse fraction and the dried fine residue were weighed, we are not presenting the weight data, because many of the samples were not completely broken up, so that the coarse fraction contained some clumped fine material. Where possible, specimens for isotope analysis were taken from a controlled size fraction, generally between 300 and 355 microns. Informa-

\footnotetext{
${ }^{1}$ Cann, J. R., Langseth, M. G., Honnorez, J., Von Herzen, R. P., White, S. M., et al., Init. Repts. DSDP, 69: Washington (U.S. Govt. Printing Office).
}

tion on the size picked is given in Table 1 . Specimens were physically cleaned by ultrasonic action first in distilled water and subsequently in analytical grade methanol. Further cleaning in a vacuum oven at $400^{\circ}$ $\mathrm{C}$ for 30 minutes preceded analysis. The carbon dioxide released by the action of $100 \%$ orthophosphoric acid was analyzed in a VG Micromass 903 mass spectrometer, and the results were calibrated by repeated analyses of various circulating carbonate standards. In general our analytical precision is about \pm 0.07 per mil.

\section{RESULTS}

All data analyzed are given in Table 1 . In a number of samples it was not possible to extract sufficient specimens of Globigerinoides ruber for analysis. In some of these we were able to analyze $G$. sacculifer instead ( $G$. ruber is considerably more abundant than $G$. sacculifer at this site, so it would not have been possible to obtain a useful record by concentrating on the latter species). In a few samples it was impossible to obtain sufficient specimens of Globigerinoides at all, and in some of these we analyzed Neogloboquadrina dutertrei instead. The result of these substitutions is that it is difficult to display the results in a manner that can be readily assimilated. Figure 1 shows the relationship between isotopic values in $G$. ruber and $G$. sacculifer in those samples in which both species were analyzed. If the only cause of variation down hole were changes in the isotopic composition of ocean water, the data points would lie on a $45^{\circ}$ straight line. The large amount of scatter and the shift of the data points above the $45^{\circ}$ line suggest that some factor (perhaps climate related) brings about a change in the isotopic variation of one or both species. Possible causes include temperature changes' affecting the water near the surface more than deeper parts of the water column, the vertical migration of deeper-dwelling species in response to density changes, and changes in the seasonal distribution of temperature or of some foraminiferal species. We do not wish to speculate further on these or other causes, but merely to point out that the problem exists (as has been known for many years). Although a correction factor can be applied to make the 
Table 1. Oxygen and carbon isotope data for Hole 504.

\begin{tabular}{|c|c|c|c|c|}
\hline $\begin{array}{l}\text { Sub-bottom } \\
\text { Depth } \\
\text { (m) }\end{array}$ & $\begin{array}{l}\text { Sample } \\
\text { (level } \\
\text { in } \mathrm{cm} \text { ) }\end{array}$ & $\delta^{18} \mathrm{O}$ & $\delta^{13} \mathrm{O}$ & $\begin{array}{l}\text { Species } \\
\text { Used (see } \\
\text { fns. a-e) }\end{array}$ \\
\hline 10.1 & $504-1-1,10$ & -0.97 & +1.07 & a \\
\hline 10.2 & $504-1-1,20$ & -0.52 & +1.65 & b \\
\hline 10.3 & $504-1-1,30$ & -0.36 & +1.94 & b \\
\hline 10.4 & $504-1-1,40$ & -1.23 & +1.93 & b \\
\hline 10.5 & $504-1-1,50$ & +0.76 & +1.82 & c \\
\hline 10.6 & $504-1-1,60$ & -1.47 & +2.13 & b \\
\hline 10.7 & $504-1-1,70$ & -1.52 & +2.22 & b \\
\hline 10.8 & $504-1-1,80$ & -1.88 & +2.12 & b \\
\hline 10.9 & $504-1-1,90$ & -1.39 & +2.09 & b \\
\hline 11.0 & $504-1-1,100$ & -1.52 & +2.13 & b \\
\hline 11.1 & $504-1-1,110$ & $\begin{array}{l}-1.22 \\
+0.12\end{array}$ & +1.95 & b \\
\hline 11.2 & $504-1-1,120$ & $\begin{array}{l}+0.12 \\
-0.03\end{array}$ & $\begin{array}{l}+1.76 \\
+1.88\end{array}$ & $\begin{array}{l}\mathrm{c} \\
\mathrm{c}\end{array}$ \\
\hline 11.4 & $504-1-1,140$ & -1.26 & +1.83 & b \\
\hline 11.5 & $504-1-2,0$ & -1.78 & +1.62 & a \\
\hline 11.6 & $504-1-2,10$ & -1.16 & +1.49 & a \\
\hline 11.7 & $504-1-2,20$ & -1.22 & +1.54 & a \\
\hline 11.8 & $504-1-2,30$ & -1.32 & +1.44 & a \\
\hline 11.9 & $504-1-2,40$ & -1.18 & +1.57 & a \\
\hline 12.4 & $504-1-2,90$ & +0.05 & +2.01 & c \\
\hline 12.5 & $504-1-2,100$ & $\begin{array}{l}-2.18 \\
-0.18\end{array}$ & $\begin{array}{l}+1.76 \\
+1.76\end{array}$ & $\begin{array}{l}\text { a } \\
\text { c }\end{array}$ \\
\hline & & -2.05 & +1.68 & $\begin{array}{l}\mathrm{c} \\
\mathrm{a}\end{array}$ \\
\hline 12.6 & $504-1-2,110$ & -1.52 & +1.85 & b \\
\hline 12.7 & $504-1-2,120$ & $\left\{\begin{array}{l}-1.41 \\
-0.79\end{array}\right.$ & $\begin{array}{l}+1.51 \\
+1.43\end{array}$ & $\begin{array}{l}a \\
b\end{array}$ \\
\hline & & $\begin{array}{l}-0.79 \\
-1.02\end{array}$ & +1.54 & $\begin{array}{l}\mathrm{b} \\
\mathrm{a}\end{array}$ \\
\hline 12.8 & $504-1-2,130$ & -0.57 & +1.77 & $\mathrm{~b}$ \\
\hline 12.9 & $504-1-2,140$ & -1.11 & +1.25 & a \\
\hline 13.0 & $504-1-2,150$ & -0.59 & +1.34 & a \\
\hline 13.1 & $504-1-3,10$ & -0.86 & +1.20 & a \\
\hline & & -0.42 & +1.86 & b \\
\hline 13.2 & $504-1-3,20$ & -0.54 & +1.68 & $\mathrm{~b}$ \\
\hline 13.3 & $504-1-3,30$ & $\begin{array}{l}-1.08 \\
-1.05\end{array}$ & $\begin{array}{l}+1.35 \\
+1.66\end{array}$ & $\mathrm{a}$ \\
\hline 13.4 & $504-1-3,40$ & $\begin{array}{l}-1.05 \\
-1.10\end{array}$ & $\begin{array}{l}+1.66 \\
+1.15\end{array}$ & $\begin{array}{l}\mathrm{b} \\
\mathrm{a}\end{array}$ \\
\hline 13.5 & $504-1-3,50$ & -1.43 & +1.23 & a \\
\hline 13.6 & $504-1-3,60$ & -1.25 & +1.17 & a \\
\hline & & $\begin{array}{l}-0.75 \\
-0.88\end{array}$ & +1.76 & b \\
\hline 13.8 & $504-1-3,80$ & $\begin{array}{l}-0.88 \\
-0.64\end{array}$ & +1.69 & $\begin{array}{l}\mathrm{a} \\
\mathrm{b}\end{array}$ \\
\hline 13.9 & $504-1-3,90$ & -0.83 & $\begin{array}{l}+1.85 \\
+1.53\end{array}$ & $\begin{array}{l}b \\
a\end{array}$ \\
\hline 14.0 & $504-1-3,100$ & -1.05 & +1.59 & a \\
\hline 14.5 & $504-2-1,10$ & -0.99 & +1.35 & d \\
\hline 14.6 & $504-2-1,20$ & -1.13 & +1.22 & a \\
\hline 14.7 & $504-2-1,30$ & -1.17 & +1.85 & d \\
\hline 14.8 & $504-2-1,40$ & -1.30 & +1.80 & d \\
\hline 14.9 & $504-2-1,50$ & -1.75 & +1.44 & a \\
\hline 15.2 & $504-2-1,80$ & -1.40 & +1.41 & a \\
\hline 15.3 & $504-2-1,90$ & -1.28 & +1.46 & a \\
\hline 15.4 & $504-2-1,100$ & -1.15 & +2.04 & d \\
\hline 15.5 & $504-2-1,110$ & -1.44 & +1.82 & d \\
\hline 15.6 & $504-2-1,120$ & -0.48 & +1.58 & b \\
\hline 15.7 & $504-2-1,130$ & -1.60 & +2.28 & b \\
\hline 15.8 & $504-2-1,140$ & -1.71 & +1.58 & a \\
\hline 15.9 & $504-2-1,150$ & -1.76 & +1.57 & a \\
\hline 16.0 & $504-2-2,10$ & -1.65 & +1.71 & a \\
\hline 16.1 & $504-2-2,20$ & $\begin{array}{l}-1.68 \\
-1.65\end{array}$ & $\begin{array}{l}+1.77 \\
+1.66\end{array}$ & $\begin{array}{l}\mathrm{b} \\
\mathrm{a}\end{array}$ \\
\hline 16.2 & $504-2-2,30$ & -1.74 & $\begin{array}{l}+1.66 \\
+1.56\end{array}$ & $\begin{array}{l}a \\
a\end{array}$ \\
\hline 16.3 & $504-2-2,40$ & -2.13 & +1.66 & a \\
\hline 16.4 & $504-2-2,50$ & -2.19 & +1.77 & a \\
\hline 16.5 & $504-2-2,60$ & -2.34 & +1.41 & a \\
\hline 16.6 & $504-2-2,70$ & -1.68 & +1.31 & a \\
\hline 16.7 & $504-2-2,80$ & -1.12 & +1.00 & a \\
\hline 16.8 & $504-2-2,90$ & -0.84 & +1.07 & a \\
\hline 16.9 & $504-2-2,100$ & -0.74 & +1.22 & a \\
\hline
\end{tabular}

Table 1. (Continued).

\begin{tabular}{|c|c|c|c|c|}
\hline $\begin{array}{l}\text { Sub-bottom } \\
\text { Depth } \\
\text { (m) }\end{array}$ & $\begin{array}{c}\text { Sample } \\
\text { (level } \\
\text { in } \mathrm{cm})\end{array}$ & $\delta^{18} \mathrm{O}$ & $\delta^{13} \mathrm{O}$ & $\begin{array}{l}\text { Species } \\
\text { Used (see } \\
\text { fns. a-e) }\end{array}$ \\
\hline 17.0 & $504-2-2,110$ & $\left\{\begin{array}{l}-0.87 \\
+1.31\end{array}\right.$ & $\begin{array}{l}+1.22 \\
+1.33\end{array}$ & $\begin{array}{l}\mathrm{a} \\
\mathrm{c}\end{array}$ \\
\hline 17.1 & $504-2-2,120$ & $\left\{\begin{array}{l}-2.02 \\
+0.17\end{array}\right.$ & $\begin{array}{l}+1.43 \\
+1.63\end{array}$ & $\begin{array}{l}\mathrm{a} \\
\mathrm{c}\end{array}$ \\
\hline 17.2 & $504-2-2,130$ & $\left\{\begin{array}{l}-0.94 \\
+1.17\end{array}\right.$ & $\begin{array}{l}+1.31 \\
+1.42\end{array}$ & $\begin{array}{l}\mathrm{a} \\
\mathrm{c}\end{array}$ \\
\hline 17.3 & $504-2-2,140$ & -1.16 & +1.31 & a \\
\hline 17.4 & $504-2-2,150$ & -0.67 & +1.67 & b \\
\hline 17.5 & $504-2-3,10$ & -0.83 & +1.62 & a \\
\hline 17.6 & $504-2-3,20$ & -0.80 & +1.41 & a \\
\hline 17.7 & $504-2-3,30$ & -0.85 & +1.76 & a \\
\hline 17.8 & $504-2-3,40$ & -1.17 & +1.62 & a \\
\hline 17.9 & $504-2-3,50$ & -1.29 & +1.78 & a \\
\hline 18.0 & $504-2-3,60$ & -1.03 & +1.75 & a \\
\hline 18.1 & $504-2-3,70$ & -1.21 & +1.58 & a \\
\hline 18.2 & $504-2-3,80$ & -1.08 & +1.81 & $\mathrm{a}$ \\
\hline 18.9 & $504-3-1,10$ & -2.21 & +2.51 & $\mathrm{~b}$ \\
\hline 19.0 & $504-3-1,20$ & $\left\{\begin{array}{l}-1.95 \\
-1.98\end{array}\right.$ & +1.88 & a \\
\hline 19.1 & $504-3-1,30$ & $\begin{array}{l}-1.98 \\
-2.13\end{array}$ & $\begin{array}{l}+2.37 \\
+1.93\end{array}$ & $\begin{array}{l}\mathrm{b} \\
\mathrm{a}\end{array}$ \\
\hline 19.2 & $504-3-1,40$ & -2.46 & +1.42 & a \\
\hline 19.3 & $504-3-1,50$ & -2.38 & $\begin{array}{l}+1.99 \\
\end{array}$ & a \\
\hline 19.4 & $504-3-1,60$ & -1.49 & +1.33 & a \\
\hline 19.5 & $504-3-1,70$ & -1.40 & +1.19 & a \\
\hline 19.6 & $504-3-1,80$ & -0.16 & +1.68 & b \\
\hline 19.7 & $504-3-1,90$ & -0.63 & +1.31 & a \\
\hline 19.8 & $504-3-1,100$ & +0.04 & +1.83 & b \\
\hline 19.9 & $504-3-1,110$ & -0.40 & +1.87 & b \\
\hline 20.0 & $504-3-1,120$ & -0.26 & +1.78 & b \\
\hline 20.1 & $504-3-1,130$ & -0.43 & +1.86 & b \\
\hline 20.2 & $504-3-1,140$ & -0.63 & +1.94 & b \\
\hline 20.4 & $504-3-2,10$ & -0.81 & +1.38 & a \\
\hline 20.5 & $504-3-2,20$ & -1.08 & +1.39 & a \\
\hline 20.6 & $504-3-2,30$ & -0.53 & +1.76 & b \\
\hline 20.7 & $504-3-2,40$ & $\left\{\begin{array}{l}-0.90 \\
-0.34\end{array}\right.$ & +1.30 & a \\
\hline 20.8 & $504-3-2,50$ & $\begin{array}{l}-0.34 \\
-0.74\end{array}$ & $\begin{array}{l}+1.96 \\
+1.76\end{array}$ & $\begin{array}{l}b \\
b\end{array}$ \\
\hline 20.9 & $504-3-2,60$ & -0.48 & +1.85 & b \\
\hline 21.0 & $504-3-2,70$ & -0.42 & +2.01 & b \\
\hline 21.1 & $504-3-2,80$ & $\left\{\begin{array}{l}-0.98 \\
-0.80\end{array}\right.$ & $\begin{array}{l}+1.55 \\
+1.71\end{array}$ & a \\
\hline 21.2 & $504-3-2,90$ & $\begin{array}{l}-0.80 \\
-1.13\end{array}$ & $\begin{array}{l}+1.71 \\
+1.58\end{array}$ & $\begin{array}{l}\mathrm{b} \\
\mathrm{a}\end{array}$ \\
\hline 21.3 & $504-3-2,100$ & -0.80 & +1.77 & a \\
\hline 21.4 & $504-3-2,110$ & -1.03 & +1.76 & a \\
\hline 21.5 & $504-3-2,120$ & -1.38 & +1.46 & a \\
\hline 21.6 & $504-3-2,130$ & -1.28 & +1.80 & c \\
\hline 21.7 & $504-3-2,140$ & -0.87 & +2.14 & b \\
\hline 21.8 & $504-3-2,150$ & -1.52 & +2.29 & b \\
\hline 21.9 & $504-3-3,10$ & $\{-1.68$ & +2.05 & a \\
\hline 22.0 & $504-3-3,20$ & $\begin{array}{l}-1.04 \\
-1.58\end{array}$ & $\begin{array}{l}+2.48 \\
+1.33\end{array}$ & $\begin{array}{l}\mathrm{b} \\
\mathrm{a}\end{array}$ \\
\hline 22.1 & $504-3-3,30$ & -1.84 & +2.22 & a \\
\hline 22.2 & $504-3-3,40$ & -1.65 & +2.25 & a \\
\hline 22.3 & $504-3-3,50$ & -1.48 & +2.20 & $\mathrm{a}$ \\
\hline 22.4 & $504-3-3,60$ & -1.46 & +2.05 & a \\
\hline 22.5 & $504-3-3,70$ & -1.17 & +2.42 & b \\
\hline 22.6 & $504-3-3,80$ & $\left\{\begin{array}{l}-1.59 \\
-1.47\end{array}\right.$ & $\begin{array}{l}+2.01 \\
+2.42\end{array}$ & a \\
\hline 22.7 & $504-3-3,90$ & $\begin{array}{l}-1.41 \\
-1.63\end{array}$ & $\begin{array}{l}+2.42 \\
+2.00\end{array}$ & a \\
\hline 22.8 & $504-3-3,100$ & -1.67 & +1.79 & a \\
\hline 22.9 & $504-3-3,110$ & -1.50 & +2.06 & a \\
\hline 23.0 & $504-3-3,120$ & -1.56 & +1.94 & a \\
\hline 23.3 & $504-4-1,10$ & -0.94 & +1.83 & b \\
\hline 23.4 & $504-4-1,20$ & -0.83 & +1.95 & b \\
\hline 23.6 & $504-4-1,40$ & -1.13 & +1.75 & a \\
\hline 23.7 & $504-4-1,50$ & -1.36 & +1.44 & a \\
\hline 23.8 & $504-4-1,60$ & -1.14 & +2.08 & b \\
\hline
\end{tabular}


Table 1. (Continued).

\begin{tabular}{|c|c|c|c|c|}
\hline $\begin{array}{l}\text { Sub-bottom } \\
\text { Depth } \\
\text { (m) }\end{array}$ & $\begin{array}{c}\text { Sample } \\
\text { (level } \\
\text { in } \mathrm{cm} \text { ) }\end{array}$ & $\delta^{18} \mathrm{O}$ & $\delta^{13} \mathrm{O}$ & $\begin{array}{l}\text { Species } \\
\text { Used (see } \\
\text { fns. a-e) }\end{array}$ \\
\hline 23.9 & $504-4-1,70$ & -1.67 & +1.47 & a \\
\hline 24.0 & $504-4-1,80$ & -1.35 & +1.88 & b \\
\hline 24.1 & $504-4-1,90$ & -1.16 & +2.18 & b \\
\hline 24.2 & $504-4-1,100$ & -1.56 & +1.87 & a \\
\hline 24.3 & $504-4-1,110$ & -1.43 & +1.32 & a \\
\hline 24.4 & $504-4-1,120$ & $\left\{\begin{array}{l}-2.02 \\
-1.38\end{array}\right.$ & $\begin{array}{l}+1.57 \\
+2.05\end{array}$ & $\begin{array}{l}\mathrm{a} \\
\mathrm{b}\end{array}$ \\
\hline 24.5 & $504-4-1,130$ & -1.85 & +1.71 & $\mathrm{a}$ \\
\hline 24.6 & $504-4-1,140$ & -1.68 & +2.16 & b \\
\hline 24.7 & $504-4-1,150$ & -1.70 & +1.30 & a \\
\hline 24.8 & $504-4-2,10$ & -1.39 & +1.54 & a \\
\hline 24.9 & $504-4-2,20$ & -1.68 & +1.59 & a \\
\hline 25.0 & $504-4-2,30$ & -1.22 & +1.50 & a \\
\hline 25.1 & $504-4-2,40$ & -1.30 & +1.97 & b \\
\hline 25.2 & $504-4-2,50$ & -1.30 & +1.35 & a \\
\hline 25.3 & $504-4-2,60$ & -1.62 & +1.91 & d \\
\hline 25.4 & $504-4-2,70$ & -1.81 & +1.68 & a \\
\hline 25.5 & $504-4-2,80$ & -1.70 & +1.70 & a \\
\hline 25.6 & $504-4-2,90$ & -1.69 & +1.77 & a \\
\hline 25.7 & $504-4-2,100$ & -0.79 & +1.50 & $\mathrm{~d}$ \\
\hline 25.8 & $504-4-2,110$ & -1.42 & +2.23 & b \\
\hline 25.9 & $504-4-2,110$ & $\begin{array}{l}-1.96 \\
-1.26\end{array}$ & $\begin{array}{l}+1.69 \\
+2.23\end{array}$ & $\begin{array}{l}a \\
b\end{array}$ \\
\hline 26.0 & $504-4-2,130$ & -2.03 & +1.66 & a \\
\hline 26.1 & $504-4-2,140$ & -1.74 & +1.53 & a \\
\hline 26.2 & $504-4-2,150$ & -1.16 & +1.35 & a \\
\hline 26.3 & $504-4-3,10$ & -1.73 & +1.46 & a \\
\hline 26.4 & $504-4-3,20$ & -0.42 & +1.41 & a \\
\hline 26.5 & $504-4-3,30$ & -0.05 & +1.34 & a \\
\hline 26.6 & $504-4-3,40$ & -0.25 & +1.31 & a \\
\hline 26.7 & $504-4-3,50$ & -0.47 & +1.20 & a \\
\hline 26.8 & $504-4-3,60$ & -0.74 & +1.10 & a \\
\hline 26.9 & $504-4-3,70$ & -0.51 & +1.30 & a \\
\hline 27.0 & $504-4-3,80$ & -0.70 & +1.37 & a \\
\hline 27.1 & $504-4-3,90$ & -0.88 & +1.23 & a \\
\hline 27.2 & $504-4-3,100$ & -0.65 & +1.34 & a \\
\hline 27.3 & $504-4-3,110$ & -1.03 & +1.02 & a \\
\hline 28.0 & $504-5-1,40$ & -1.91 & +1.71 & a \\
\hline 28.1 & $504-5-1,50$ & -1.51 & +1.80 & a \\
\hline 28.2 & $504-5-1,60$ & -1.48 & +1.57 & a \\
\hline 28.3 & $504-5-1,70$ & -1.25 & +1.54 & a \\
\hline 28.4 & $504-5-1,80$ & -1.24 & +1.57 & a \\
\hline 28.5 & $504-5-1,90$ & -1.48 & +1.54 & $\mathbf{a}$ \\
\hline 28.6 & $504-5-1,100$ & -1.15 & +1.27 & a \\
\hline 28.7 & $504-5-1,110$ & -0.84 & +1.20 & a \\
\hline 28.8 & $504-5-1,120$ & -0.57 & +1.24 & a \\
\hline 28.9 & $504-5-1,130$ & -0.77 & +1.19 & a \\
\hline 29.0 & $504-5-1,140$ & -0.99 & +1.37 & a \\
\hline 29.2 & $504-5-2,10$ & -1.18 & +1.59 & a \\
\hline 29.3 & $504-5-2,20$ & -1.40 & +1.52 & a \\
\hline 29.4 & $504-5-2,30$ & -1.37 & +1.49 & a \\
\hline 29.5 & $504-5-2,40$ & -1.41 & +1.39 & a \\
\hline 29.6 & $504-5-2,50$ & -1.11 & +1.43 & a \\
\hline 29.7 & $504-5-2,60$ & -1.11 & +1.55 & a \\
\hline 29.8 & $504-5-2,70$ & -1.14 & +1.27 & a \\
\hline 29.9 & $504-5-2,80$ & -1.08 & +1.33 & a \\
\hline 30.0 & $504-5-2,90$ & -1.15 & +0.99 & a \\
\hline 30.1 & $504-5-2,100$ & -0.91 & +1.41 & a \\
\hline 30.2 & $504-5-2,110$ & -1.10 & +1.43 & a \\
\hline 30.3 & $504-5-2,120$ & -0.95 & +1.31 & a \\
\hline 30.4 & $504-5-2,130$ & -1.25 & +1.22 & a \\
\hline 30.5 & $504-5-2,140$ & -1.45 & +1.39 & a \\
\hline 30.6 & $504-5-2,150$ & -1.38 & +2.06 & b \\
\hline 30.7 & $504-5-3,10$ & -1.45 & +1.34 & a \\
\hline 30.8 & $504-5-3,20$ & -1.37 & +1.97 & b \\
\hline 30.9 & $504-5-3,30$ & -1.28 & +1.52 & d \\
\hline 31.0 & $504-5-3,40$ & -1.67 & +1.53 & a \\
\hline 32.3 & $504-6-1,30$ & -1.16 & +1.46 & a \\
\hline
\end{tabular}

Table 1. (Continued).

\begin{tabular}{|c|c|c|c|c|}
\hline $\begin{array}{l}\text { Sub-bottom } \\
\text { Depth } \\
\text { (m) }\end{array}$ & $\begin{array}{c}\text { Sample } \\
\text { (level } \\
\text { in } \mathrm{cm} \text { ) }\end{array}$ & $\delta^{18} \mathrm{O}$ & $\delta^{13} \mathrm{O}$ & $\begin{array}{l}\text { Species } \\
\text { Used (see } \\
\text { fns. a-e) }\end{array}$ \\
\hline 32.5 & $504-6-1,50$ & -1.63 & +1.32 & a \\
\hline 32.6 & $504-6-1,60$ & -1.37 & +1.46 & a \\
\hline 32.7 & $504-6-1,70$ & -1.47 & +1.39 & a \\
\hline 32.8 & $504-6-1,80$ & -1.32 & +1.32 & a \\
\hline 32.9 & $504-6-1,90$ & -1.60 & +1.24 & a \\
\hline 33.0 & $504-6-1,100$ & -1.91 & +1.32 & a \\
\hline 33.1 & $504-6-1,110$ & -1.57 & +1.38 & a \\
\hline 33.2 & $504-6-1,120$ & -1.72 & +1.42 & a \\
\hline 33.3 & $504-6-1,130$ & -1.40 & +1.58 & a \\
\hline 33.4 & $504-6-1,140$ & -1.64 & +1.65 & a \\
\hline 33.5 & $504-6-1,150$ & -1.67 & +1.31 & a \\
\hline 33.6 & $504-6-2,10$ & -1.89 & +1.37 & a \\
\hline 33.7 & $504-6-2,20$ & -1.86 & +1.33 & a \\
\hline 33.8 & $504-6-2,30$ & -1.65 & +1.55 & a \\
\hline 33.9 & $504-6-2,40$ & -1.45 & +1.46 & a \\
\hline 34.0 & $504-6-2,50$ & -1.73 & +1.45 & a \\
\hline 34.1 & $504-6-2,60$ & -1.67 & +1.44 & a \\
\hline 34.2 & $504-6-2,70$ & -1.80 & +1.20 & a \\
\hline 34.3 & $504-6-2,80$ & -1.63 & +1.26 & a \\
\hline 34.4 & $504-6-2,90$ & -0.97 & +0.98 & a \\
\hline 34.5 & $504-6-2,100$ & -0.53 & +0.91 & a \\
\hline 34.6 & $504-6-2,110$ & -0.21 & +1.11 & a \\
\hline 34.7 & $504-6-2,120$ & -0.14 & +0.96 & a \\
\hline 34.8 & $504-6-2,130$ & -0.23 & +0.95 & a \\
\hline 34.9 & $504-6-2,140$ & -0.34 & +1.01 & a \\
\hline 35.0 & $504-6-2,150$ & -0.86 & +1.23 & a \\
\hline 35.1 & $504-6-3,10$ & -0.48 & +1.12 & a \\
\hline 35.2 & $504-6-3,20$ & -0.52 & +1.21 & a \\
\hline 35.3 & $504-6-3,30$ & -0.46 & +1.26 & a \\
\hline 35.4 & $504-6-3,40$ & -0.53 & +1.17 & a \\
\hline 35.5 & $504-6-3,50$ & -0.39 & +1.06 & a \\
\hline 35.6 & $504-6-3,60$ & -0.25 & +1.38 & b \\
\hline 35.7 & $504-6-3,70$ & -0.67 & +1.08 & a \\
\hline 35.8 & $504-6-3,80$ & -0.98 & +1.09 & a \\
\hline 35.9 & $504-6-3,90$ & -1.02 & +1.19 & a \\
\hline 36.0 & $504-6-3,100$ & -1.12 & +1.26 & a \\
\hline 36.1 & $504-6-3,110$ & -1.17 & +1.21 & a \\
\hline 36.2 & $504-6-3,120$ & -1.32 & +1.01 & a \\
\hline 36.5 & $504-7-1,10$ & -0.47 & +1.50 & b \\
\hline 36.7 & $504-7-1,30$ & -1.56 & +1.89 & d \\
\hline 36.8 & $504-7-1,40$ & $\left\{\begin{array}{l}-1.51 \\
-1.17\end{array}\right.$ & +1.73 & a \\
\hline 36.9 & $504-7-1,50$ & $\begin{array}{l}-1.17 \\
-1.70\end{array}$ & +2.37 & b \\
\hline 37.0 & $504-7-1,60$ & & +1.53 & a \\
\hline 37.1 & $504-7-1,70$ & & +1.68 & a \\
\hline 37.3 & $504-7-1,90$ & -1.92 & +1.59 & a \\
\hline 37.4 & $504-7-1,100$ & -2.01 & +1.58 & a \\
\hline 37.5 & $504-7-1,110$ & -1.97 & +1.51 & a \\
\hline 37.6 & $\begin{array}{l}504-7-1,110 \\
504-7-1,120\end{array}$ & -1.38 & +1.42 & a \\
\hline & & -1.24 & +1.43 & a \\
\hline $\begin{array}{l}31.1 \\
37.8\end{array}$ & $504-7-1,130$ & -0.95 & +1.32 & a \\
\hline 37.8 & $504-7-1,140$ & -1.19 & +1.60 & a \\
\hline 37.9 & $504-7-2,0$ & -1.23 & +1.23 & a \\
\hline 38.0 & $504-7-2,10$ & -1.41 & +1.56 & a \\
\hline 38.1 & $504-7-2,20$ & $\begin{array}{l}-1.17 \\
-1.20\end{array}$ & $\begin{array}{l}+1.77 \\
+2.13\end{array}$ & a \\
\hline 38.2 & $504-7-2,30$ & $\begin{array}{l}-1.20 \\
-1.51\end{array}$ & $\begin{array}{l}+2.13 \\
+1.71\end{array}$ & $\begin{array}{l}\mathrm{b} \\
\mathrm{a}\end{array}$ \\
\hline 38.3 & $504-7-2,40$ & -1.96 & +1.75 & a \\
\hline 38.4 & $504-7-2,50$ & +1.72 & +1.61 & a \\
\hline 38.5 & $504-7-2,60$ & -1.41 & +1.54 & a \\
\hline 38.6 & $504-7-2,70$ & -1.41 & +1.65 & a \\
\hline 38.7 & $504-7-2,80$ & -1.48 & +1.70 & a \\
\hline 38.9 & $504-7-2,100$ & $\left\{\begin{array}{l}-1.15 \\
-0.77\end{array}\right.$ & $\begin{array}{l}+1.60 \\
+1.84\end{array}$ & $\begin{array}{l}\mathrm{a} \\
\mathrm{b}\end{array}$ \\
\hline 39.0 & $504-7-2,110$ & -1.18 & +1.63 & $\mathrm{a}$ \\
\hline & $304-1-2,110$ & -1.23 & +2.06 & b \\
\hline 39.1 & $504-7-2,120$ & -1.53 & +1.73 & a \\
\hline 39.2 & $504-7-2,130$ & -1.48 & +1.69 & a \\
\hline 39.3 & $504-7-2,140$ & -1.53 & +1.53 & a \\
\hline
\end{tabular}


Table 1. (Continued).

\begin{tabular}{|c|c|c|c|c|}
\hline $\begin{array}{l}\text { Sub-bottom } \\
\text { Depth } \\
\text { (m) }\end{array}$ & $\begin{array}{c}\text { Sample } \\
\text { (level } \\
\text { in } \mathrm{cm} \text { ) }\end{array}$ & $\delta^{18} \mathrm{O}$ & $\delta^{13} \mathrm{O}$ & $\begin{array}{l}\text { Species } \\
\text { Used (see } \\
\text { fns. a-e) }\end{array}$ \\
\hline 39.4 & $504-7-3,10$ & -1.08 & +1.64 & a \\
\hline 39.6 & $504-7-3,30$ & -1.02 & +1.45 & a \\
\hline 39.7 & $504-7-3,40$ & -1.07 & +1.31 & a \\
\hline 39.8 & $504-7-3,50$ & -1.21 & +1.61 & a \\
\hline 39.9 & $504-7-3,60$ & -1.72 & +1.36 & a \\
\hline 40.0 & $504-7-3,70$ & -1.73 & +1.76 & a \\
\hline 40.1 & $504-7-3,80$ & -1.67 & +1.51 & a \\
\hline 40.3 & $504-7-3,100$ & -1.68 & +1.69 & a \\
\hline 40.4 & $504-7-3,110$ & -1.38 & +1.48 & a \\
\hline 40.5 & $504-7-3,120$ & -1.59 & +1.46 & a \\
\hline 40.6 & $504-7-3,130$ & -0.99 & +1.28 & a \\
\hline 40.7 & $504-7-3,140$ & -1.09 & +1.22 & a \\
\hline 40.8 & $504-7-3,150$ & -1.00 & +1.18 & a \\
\hline 41.0 & $504-8-1,20$ & -1.39 & +1.51 & a \\
\hline 41.1 & $504-8-1,30$ & -1.31 & +1.15 & a \\
\hline 41.2 & $504-8-1,40$ & -1.46 & +1.39 & a \\
\hline 41.3 & $504-8-1,50$ & -1.38 & +1.01 & a \\
\hline 41.4 & $504-8-1,60$ & -1.55 & +0.93 & a \\
\hline 41.5 & $504-8-1,70$ & -1.71 & +1.07 & a \\
\hline 41.6 & $504-8-1,80$ & -1.43 & +1.39 & a \\
\hline 41.8 & $504-8-1,100$ & -1.19 & +1.67 & b \\
\hline 41.9 & $504-8-1,110$ & -1.10 & +1.92 & b \\
\hline 42.0 & $504-8-1,120$ & -1.64 & +1.28 & a \\
\hline 42.1 & $504-8-1,130$ & -1.92 & +1.63 & a \\
\hline 42.2 & $504-8-1,140$ & -2.13 & +1.84 & a \\
\hline 42.3 & $504-8-1,150$ & -1.85 & +1.37 & a \\
\hline 42.4 & $504-8-2,10$ & -2.21 & +1.59 & a \\
\hline 42.5 & $504-8-2,20$ & -1.71 & +1.79 & a \\
\hline 42.6 & $504-8-2,30$ & -1.86 & +1.46 & a \\
\hline 42.7 & $504-8-2,40$ & -1.49 & +1.62 & a \\
\hline 42.8 & $504-8-2,50$ & -1.75 & +1.69 & a \\
\hline 42.9 & $504-8-2,60$ & $\begin{array}{l}-1.53 \\
-0.65\end{array}$ & +1.42 & $\begin{array}{l}a \\
b\end{array}$ \\
\hline 43.0 & $504-8-2,70$ & $\begin{array}{l}-0.65 \\
-1.37\end{array}$ & $\begin{array}{l}+1.54 \\
+1.62\end{array}$ & a \\
\hline 43.1 & $504-8-2,80$ & -1.43 & +1.72 & a \\
\hline 43.2 & $504-8-2,90$ & -1.58 & +1.58 & a \\
\hline 43.3 & $504-8-2,100$ & -1.23 & +1.64 & b \\
\hline 43.4 & $504-8-2,110$ & -1.52 & +1.29 & a \\
\hline 43.8 & $504-8-3,0$ & -1.48 & +1.62 & a \\
\hline 43.9 & $504-8-3,10$ & -1.68 & +1.38 & a \\
\hline 44.0 & $504-8-3,20$ & -1.54 & +1.60 & a \\
\hline 44.1 & $504-8-3,30$ & -1.56 & +1.55 & a \\
\hline 44.2 & $504-8-3,40$ & -1.62 & +1.34 & a \\
\hline 44.3 & $504-8-3,50$ & -1.37 & +1.10 & a \\
\hline 44.4 & $504-8-3,60$ & -1.37 & +0.96 & a \\
\hline 44.5 & $504-8-3,70$ & -1.28 & +1.18 & a \\
\hline 44.6 & $504-8-3,80$ & -1.21 & +0.93 & a \\
\hline 44.7 & $504-8-3,90$ & -0.92 & +0.96 & a \\
\hline 44.8 & $504-8-3,100$ & -1.05 & +0.80 & a \\
\hline 44.9 & $504-8-3,110$ & -1.08 & +1.11 & a \\
\hline 45.0 & $504-8-3,120$ & -1.32 & +0.82 & a \\
\hline 45.8 & $504-9-1,60$ & -1.15 & +1.04 & a \\
\hline 45.9 & $504-9-1,70$ & -1.45 & +1.34 & a \\
\hline 46.0 & $504-9-1,80$ & -1.48 & +1.12 & a \\
\hline 46.3 & $504-9-1,110$ & -1.68 & +1.38 & a \\
\hline 46.4 & $504-9-1,120$ & -1.87 & +1.43 & a \\
\hline 46.5 & $504-9-1,130$ & -1.49 & +1.96 & a \\
\hline 46.6 & $504-9-1,140$ & -1.54 & +1.94 & a \\
\hline 46.8 & $504-9-2,10$ & -1.80 & +1.90 & a \\
\hline 46.9 & $504-9-2,20$ & -1.77 & +2.00 & a \\
\hline 47.0 & $504-9-2,30$ & -2.17 & +1.90 & a \\
\hline 47.1 & $504-9-2,40$ & -1.91 & +1.55 & a \\
\hline 47.2 & $504-9-2,50$ & -1.84 & +1.77 & a \\
\hline 47.3 & $504-9-2,60$ & -1.35 & +1.77 & a \\
\hline 47.4 & $504-9-2,70$ & -1.39 & +1.69 & a \\
\hline 47.5 & $504-9-2,80$ & -1.41 & +1.48 & a \\
\hline 47.6 & $504-9-2,90$ & -1.32 & +1.35 & a \\
\hline 47.7 & $504-9-2,100$ & -1.49 & +1.52 & a \\
\hline
\end{tabular}

Table 1. (Continued).

\begin{tabular}{|c|c|c|c|c|}
\hline $\begin{array}{l}\text { Sub-bottom } \\
\text { Depth } \\
\text { (m) }\end{array}$ & $\begin{array}{c}\text { Sample } \\
\text { (level } \\
\text { in } \mathrm{cm} \text { ) }\end{array}$ & $\delta^{18} \mathrm{O}$ & $\delta^{13} \mathrm{O}$ & $\begin{array}{l}\text { Species } \\
\text { Used (see } \\
\text { fns. a-e) }\end{array}$ \\
\hline 47.8 & $504-9-2,110$ & -1.33 & +1.50 & a \\
\hline 47.9 & $504-9-2,120$ & -1.54 & +1.51 & a \\
\hline 48.0 & $504-9-2,130$ & -1.62 & +1.48 & a \\
\hline 48.1 & $504-9-2,140$ & -1.44 & +1.25 & a \\
\hline 48.2 & $504-9-2,150$ & -1.16 & +1.36 & a \\
\hline 48.3 & $504-9-3,10$ & -1.12 & +1.29 & a \\
\hline 48.4 & $504-9-3,20$ & -1.10 & +1.24 & a \\
\hline 48.5 & $504-9-3,30$ & -1.15 & +1.13 & a \\
\hline 48.6 & $504-9-3,40$ & -1.18 & +1.30 & a \\
\hline 48.7 & $504-9-3,50$ & -1.12 & +1.30 & a \\
\hline 48.8 & $504-9-3,60$ & -1.13 & +1.41 & $\mathrm{a}$ \\
\hline 48.9 & $504-9-3,70$ & -1.42 & +1.20 & a \\
\hline 49.0 & $504-9-3,80$ & -1.32 & +1.09 & a \\
\hline 49.1 & $504-9-3,90$ & -1.16 & +1.38 & a \\
\hline 49.2 & $504-9-3,100$ & -1.75 & +1.24 & a \\
\hline 49.3 & $504-9-3,110$ & -1.55 & +1.46 & a \\
\hline 49.4 & $504-9-3,120$ & -1.53 & +1.32 & a \\
\hline 50.6 & $504-10-3,100$ & -1.70 & +1.72 & a \\
\hline 50.7 & $504-10-3,110$ & -1.86 & +1.52 & a \\
\hline 50.8 & $504-10-3,120$ & -1.56 & +1.38 & a \\
\hline 50.9 & $504-10-3,130$ & -1.37 & +1.15 & a \\
\hline 51.0 & $504-10-3,140$ & -0.87 & +1.09 & a \\
\hline 51.1 & $504-10-3,150$ & -0.97 & +0.98 & a \\
\hline 51.2 & $504-10-2,10$ & -0.77 & +1.01 & a \\
\hline 51.3 & $504-10-2,20$ & -0.81 & +1.17 & a \\
\hline 51.4 & $504-10-2,30$ & -1.05 & +1.08 & a \\
\hline 51.5 & $504-10-2,40$ & -1.45 & +1.25 & a \\
\hline 51.6 & $504-10-2,50$ & -1.49 & +1.31 & a \\
\hline 51.7 & $504-10-2,60$ & -1.72 & +1.54 & a \\
\hline 51.8 & $504-10-2,70$ & -1.42 & +1.51 & a \\
\hline 51.9 & $504-10-2,80$ & -1.48 & +1.48 & a \\
\hline 52.0 & $504-10-2,90$ & -1.51 & +1.65 & a \\
\hline 52.1 & $504-10-2,100$ & -1.50 & +1.79 & a \\
\hline 52.2 & $504-10-2,110$ & -1.74 & +1.53 & a \\
\hline 52.6 & $504-10-3,0^{*}$ & -1.13 & +1.04 & a \\
\hline 52.7 & $504-10-3,10$ & -1.22 & +1.17 & a \\
\hline 52.8 & $504-10-3,20$ & -1.28 & +0.70 & a \\
\hline 52.9 & $504-10-3,30$ & -1.08 & +1.10 & a \\
\hline 53.0 & $504-10-3,40$ & -0.91 & +1.29 & a \\
\hline 53.1 & $504-10-3,50$ & -0.76 & +1.84 & b \\
\hline 53.2 & $504-10-3,60$ & -1.29 & +1.45 & a \\
\hline 53.3 & $504-10-3,70$ & -1.37 & +1.32 & a \\
\hline 54.2 & $504-11-1,20$ & -1.44 & +1.53 & a \\
\hline 54.3 & $504-11-1,30$ & -1.63 & +1.38 & a \\
\hline 54.4 & $504-11-1,40$ & -1.30 & +1.31 & a \\
\hline 54.5 & $504-11-1,50$ & -1.18 & +1.32 & a \\
\hline 54.6 & $504-11-1,60$ & -1.07 & +1.13 & a \\
\hline 54.7 & $504-11-1,70$ & -1.25 & +0.95 & a \\
\hline 54.8 & $504-11-1,80$ & -1.13 & +1.14 & a \\
\hline 54.9 & $504-11-1,90$ & -1.62 & +0.90 & a \\
\hline 55.0 & $504-11-1,100$ & -1.48 & +1.15 & a \\
\hline 55.1 & $504-11-1,110$ & -1.39 & +1.03 & a \\
\hline 55.2 & $504-11-1,120$ & -1.40 & +1.13 & a \\
\hline 55.3 & $504-11-1,130$ & -1.45 & +1.25 & a \\
\hline 55.4 & $504-11-1,140$ & -1.26 & +1.02 & a \\
\hline 55.5 & $504-11-2,0$ & -1.62 & +1.52 & a \\
\hline 55.6 & $504-11-2,10$ & -1.97 & +1.54 & a \\
\hline 55.7 & $504-11-2,20$ & -1.76 & +1.60 & a \\
\hline 55.8 & $504-11-2,30$ & -1.73 & +1.50 & a \\
\hline 55.9 & $504-11-2,40$ & -1.40 & +1.28 & a \\
\hline 56.0 & $504-11-2,50$ & -1.39 & +1.22 & a \\
\hline 56.1 & $504-11-2,60$ & -1.52 & +1.04 & a \\
\hline 56.2 & $504-11-2,70$ & -0.99 & +1.03 & a \\
\hline 56.3 & $504-11-2,80$ & -1.08 & +1.07 & a \\
\hline 56.4 & $504-11-2,90$ & -1.15 & +1.20 & a \\
\hline 56.5 & $504-11-2,100$ & -1.55 & +1.31 & a \\
\hline 56.6 & $504-11-2,110$ & -1.29 & +1.23 & a \\
\hline 56.7 & $504-11-2,120$ & -1.48 & +1.46 & a \\
\hline
\end{tabular}


Table 1. (Continued).

\begin{tabular}{|c|c|c|c|c|}
\hline $\begin{array}{l}\text { Sub-bottom } \\
\text { Depth } \\
\text { (m) }\end{array}$ & $\begin{array}{c}\text { Sample } \\
\text { (level } \\
\text { in } \mathrm{cm} \text { ) }\end{array}$ & $\delta^{18} \mathrm{O}$ & $\delta^{13} \mathrm{O}$ & $\begin{array}{l}\text { Species } \\
\text { Used (see } \\
\text { fns. a-e) }\end{array}$ \\
\hline 56.8 & $504-11-2,130$ & $\left\{\begin{array}{l}-1.65 \\
-1.43\end{array}\right.$ & $\begin{array}{l}+1.13 \\
+1.82\end{array}$ & $\begin{array}{l}a \\
b\end{array}$ \\
\hline 56.9 & $504-11-2,140$ & -1.22 & +1.81 & a \\
\hline 57.0 & $504-11-2,150$ & -1.48 & +1.36 & a \\
\hline 57.1 & $504-11-3,10$ & -1.78 & +1.35 & a \\
\hline 57.2 & $504-11-3,20$ & -1.84 & +1.20 & a \\
\hline 57.3 & $504-11-3,30$ & -1.62 & +1.54 & a \\
\hline 57.4 & $504-11-3,40$ & -1.64 & +1.41 & a \\
\hline 57.5 & $504-11-3,50$ & -1.42 & +1.15 & a \\
\hline 57.6 & $504-11-3,60$ & -1.53 & +1.03 & a \\
\hline 57.7 & $504-11-3,70$ & -1.14 & +1.30 & a \\
\hline 57.8 & $504-11-2,80$ & -1.27 & +1.04 & a \\
\hline 57.9 & $504-11-3,90$ & -1.26 & +0.89 & a \\
\hline 58.0 & $504-11-3,100$ & -1.37 & +1.05 & a \\
\hline 58.1 & $504-11-2,110$ & -1.14 & +0.78 & a \\
\hline 58.5 & $504-12-1,10$ & -1.73 & +1.46 & a \\
\hline 58.6 & $504-12-1,20$ & -1.64 & +1.45 & a \\
\hline 58.7 & $504-12-1,30$ & -1.80 & +1.31 & a \\
\hline 58.8 & $504-12-1,40$ & -1.67 & +1.38 & a \\
\hline 58.9 & $504-12-1,50$ & -1.57 & +1.39 & a \\
\hline 59.5 & $504-12-1,110$ & -1.85 & +1.54 & a \\
\hline 59.6 & $504-12-1,120$ & -1.37 & +0.96 & a \\
\hline 59.7 & $504-12-1,130$ & -1.12 & +0.94 & a \\
\hline 59.8 & $504-12-1,140$ & -1.10 & +0.88 & a \\
\hline 59.9 & $504-12-2,0$ & -1.31 & +0.88 & a \\
\hline 60.1 & $504-12-2,20$ & -1.57 & +1.06 & a \\
\hline 60.2 & $504-12-2,30$ & -1.69 & +1.17 & a \\
\hline 60.3 & $504-12-2,40$ & -1.77 & +1.38 & a \\
\hline 60.4 & $504-12-2,50$ & -1.83 & +1.54 & a \\
\hline 60.5 & $504-12-2,60$ & -1.85 & +1.46 & a \\
\hline 60.6 & $504-12-2,70$ & -1.51 & +1.54 & a \\
\hline 60.7 & $504-12-2,80$ & -1.90 & +1.46 & a \\
\hline 60.8 & $504-12-2,90$ & -1.65 & +1.29 & a \\
\hline 60.9 & $504-12-2,100$ & -1.64 & +1.53 & a \\
\hline 61.0 & $504-12-2,110$ & -1.87 & +1.48 & a \\
\hline 61.4 & $504-12-3,0$ & -1.45 & +1.15 & a \\
\hline 61.5 & $504-12-3,10$ & -1.40 & +1.31 & a \\
\hline 61.6 & $504-12-3,20$ & -1.66 & +1.18 & a \\
\hline 61.8 & $504-12-3,40$ & -1.61 & +1.67 & a \\
\hline 61.9 & $504-12-3,50$ & -1.79 & +1.39 & a \\
\hline 62.0 & $504-12-3,60$ & -1.63 & +1.24 & a \\
\hline 62.1 & $504-12-3,70$ & -1.29 & +1.33 & a \\
\hline 62.2 & $504-12-3,80$ & -1.20 & +0.99 & a \\
\hline 62.3 & $504-12-3,90$ & -1.17 & +0.89 & a \\
\hline 62.4 & $504-12-3,100$ & -1.17 & +0.83 & a \\
\hline 62.5 & $504-12-3,110$ & -1.08 & +1.10 & a \\
\hline 62.6 & $504-12-3,120$ & -1.30 & +1.02 & a \\
\hline 62.9 & $504-13-1,10$ & -1.62 & +1.52 & a \\
\hline 63.2 & $504-13-1,40$ & -1.67 & +1.22 & a \\
\hline 63.3 & $504-13-1,50$ & -1.53 & +1.37 & a \\
\hline 63.4 & $504-13-1,60$ & -1.29 & +0.84 & a \\
\hline 63.5 & $504-13-1,70$ & -1.17 & +1.01 & a \\
\hline 63.6 & $504-13-1,80$ & -0.92 & +1.05 & a \\
\hline 63.7 & $504-13-1,90$ & -1.07 & +1.03 & b \\
\hline 63.8 & $504-13-1,100$ & -1.52 & +1.29 & a \\
\hline 63.9 & $504-13-1,110$ & -1.40 & +0.93 & a \\
\hline 64.0 & $504-13-1,120$ & -1.53 & +1.09 & a \\
\hline 64.1 & $504-13-1,130$ & -1.46 & +1.38 & a \\
\hline 64.2 & $504-13-1,140$ & -1.58 & +1.33 & a \\
\hline 64.3 & $504-13-1,150$ & -1.42 & +1.52 & a \\
\hline 64.4 & $504-13-2,10$ & -1.59 & +1.38 & a \\
\hline 64.5 & $504-13-2,20$ & -1.72 & +1.40 & a \\
\hline 64.6 & $504-13-2,30$ & -1.55 & +1.14 & a \\
\hline 64.7 & $504-13-2,40$ & -1.46 & +1.37 & a \\
\hline 64.8 & $504-13-2,50$ & -1.54 & +1.41 & a \\
\hline 64.9 & $504-13-2,60$ & -1.67 & +1.69 & a \\
\hline 65.0 & $504-13-2,70$ & -1.42 & +1.29 & a \\
\hline 65.1 & $504-13-2,80$ & -1.51 & +1.27 & a \\
\hline
\end{tabular}

Table 1. (Continued).

\begin{tabular}{|c|c|c|c|c|}
\hline $\begin{array}{l}\text { Sub-bottom } \\
\text { Depth } \\
\text { (m) }\end{array}$ & $\begin{array}{c}\text { Sample } \\
\text { (level } \\
\text { in } \mathrm{cm} \text { ) }\end{array}$ & $\delta^{18} \mathrm{O}$ & $\delta^{13} \mathrm{O}$ & $\begin{array}{l}\text { Species } \\
\text { Used (see } \\
\text { fns. a-e) }\end{array}$ \\
\hline 65.2 & $504-13-2,90$ & -1.55 & +1.26 & a \\
\hline 65.3 & $504-13-2,100$ & -1.59 & +1.34 & a \\
\hline 65.4 & $504-13-2,110$ & -1.58 & +1.54 & d \\
\hline 65.5 & $504-13-2,120$ & -1.30 & +1.42 & d \\
\hline 65.6 & $504-13-2,130$ & -1.30 & +1.04 & d \\
\hline 65.7 & $504-13-2,140$ & -1.37 & +1.21 & a \\
\hline 65.8 & $504-13-2,150$ & -1.57 & +0.89 & a \\
\hline 65.9 & $504-13-3,10$ & -1.17 & +0.99 & a \\
\hline 66.0 & $504-13-3,20$ & -1.16 & +0.74 & a \\
\hline 66.1 & $504-13-3,30$ & -1.07 & +0.86 & a \\
\hline 66.2 & $504-13-3,40$ & -1.05 & +0.70 & a \\
\hline 66.3 & $504-13-3,50$ & -0.73 & +1.12 & $\mathrm{a}$ \\
\hline 66.4 & $504-13-3,60$ & -1.19 & +0.49 & a \\
\hline 66.5 & $504-13-3,70$ & -1.26 & +1.01 & a \\
\hline 66.6 & $504-13-3,80$ & -0.93 & +0.67 & a \\
\hline 68.1 & $504-14-1,90$ & -1.84 & +0.93 & a \\
\hline 68.2 & $504-14-1,100$ & -1.77 & +0.93 & a \\
\hline 68.3 & $504-14-1,110$ & -1.63 & +1.06 & a \\
\hline 68.4 & $504-14-1,120$ & -1.67 & +0.87 & a \\
\hline 68.5 & $504-14-1,130$ & -1.52 & +0.98 & a \\
\hline 68.6 & $504-14-1,140$ & -1.41 & +0.69 & a \\
\hline 68.7 & $504-14-1,150$ & -1.54 & +1.12 & a \\
\hline 68.8 & $504-14-1,10$ & -1.82 & +1.07 & a \\
\hline 68.9 & $504-14-1,20$ & -1.73 & +1.20 & a \\
\hline 69.0 & $504-14-1,30$ & -1.43 & +1.02 & a \\
\hline 69.1 & $504-14-1,40$ & -1.60 & +0.84 & a \\
\hline 69.2 & $504-14-1,50$ & -1.22 & +0.54 & a \\
\hline 69.4 & $504-14-1,70$ & -1.53 & +0.54 & a \\
\hline 70.3 & $504-14-2,10$ & -1.73 & +1.04 & a \\
\hline 70.4 & $504-14-2,20$ & -1.51 & +0.58 & a \\
\hline 70.5 & $504-14-2,30$ & -1.27 & +0.48 & a \\
\hline 70.6 & $504-14-2,40$ & -1.26 & +0.19 & a \\
\hline 70.7 & $504-14-2,50$ & -1.22 & +0.02 & a \\
\hline 70.8 & $504-14-2,60$ & -1.37 & +0.30 & a \\
\hline 70.9 & $504-14-2,70$ & -1.45 & +0.40 & a \\
\hline 71.0 & $504-14-2,80$ & -1.47 & +0.30 & a \\
\hline 71.1 & $504-14-2,90$ & -1.37 & +0.30 & a \\
\hline 71.2 & $504-14-2,100$ & -1.16 & +0.42 & a \\
\hline 71.3 & $504-14-2,110$ & -1.20 & +0.85 & a \\
\hline 71.5 & $504-14-2,130$ & -1.03 & +0.67 & a \\
\hline 71.7 & $504-15-1,10$ & -1.69 & +1.14 & a \\
\hline 71.8 & $504-15-1,20$ & -1.95 & +0.90 & a \\
\hline 71.9 & $504-15-1,30$ & -1.76 & +0.82 & a \\
\hline 72.0 & $504-15-1,40$ & -1.38 & +0.68 & a \\
\hline 72.2 & $504-15-1,60$ & -0.72 & +0.89 & d \\
\hline 72.8 & $504-15-1,120$ & -1.77 & +1.11 & a \\
\hline 73.0 & $504-15-1,140$ & -1.36 & +1.43 & b \\
\hline 73.1 & $504-15-1,150$ & -1.45 & +0.91 & d \\
\hline 73.2 & $504-15-1,10$ & -1.53 & +0.92 & a \\
\hline 73.8 & $504-15-1,70$ & -1.36 & +1.82 & b \\
\hline 73.9 & $504-15-1,80$ & -1.75 & +1.13 & a \\
\hline 74.0 & $504-15-1,90$ & -1.44 & +1.29 & a \\
\hline 74.1 & $504-15-1,100$ & -1.70 & +1.37 & a \\
\hline 74.2 & $504-15-1,110$ & -1.37 & +0.99 & d \\
\hline 74.3 & $504-15-1,120$ & -1.50 & +1.08 & a \\
\hline 74.4 & $504-15-2,130$ & -1.67 & +0.90 & a \\
\hline 74.5 & $504-15-2,140$ & -1.46 & +1.31 & d \\
\hline 74.6 & $504-15-3,0$ & -1.61 & +0.69 & a \\
\hline 74.7 & $504-15-3,10$ & -1.38 & +1.05 & d \\
\hline 74.8 & $504-15-3,20$ & -1.10 & +0.77 & d \\
\hline 74.9 & $504-15-3,30$ & -1.71 & +0.91 & d \\
\hline 75.0 & $504-15-3,40$ & -1.43 & +1.15 & d \\
\hline 75.1 & $504-15-3,50$ & -1.47 & +0.43 & a \\
\hline 75.2 & $504-15-3,60$ & -1.29 & +0.62 & a \\
\hline 75.3 & $504-15-3,70$ & -1.63 & +1.14 & d \\
\hline 75.6 & $504-15-3,100$ & -1.42 & +0.48 & a \\
\hline 75.7 & $504-15-3,110$ & -1.63 & +0.93 & a \\
\hline 76.1 & $504-16-1,10$ & -1.77 & +0.69 & a \\
\hline
\end{tabular}


Table 1. (Continued).

\begin{tabular}{|c|c|c|c|c|}
\hline $\begin{array}{l}\text { Sub-bottom } \\
\text { Depth } \\
\text { (m) }\end{array}$ & $\begin{array}{c}\text { Sample } \\
\text { (level } \\
\text { in } \mathrm{cm} \text { ) }\end{array}$ & $\delta^{18} \mathrm{O}$ & $\delta^{13} \mathrm{O}$ & $\begin{array}{l}\text { Species } \\
\text { Used (see } \\
\text { fns. a-e) }\end{array}$ \\
\hline 76.2 & $504-16-1,20$ & -1.49 & +0.74 & a \\
\hline 76.4 & $504-16-1,40$ & -1.58 & +0.64 & a \\
\hline 76.5 & $504-16-1,50$ & -0.94 & +1.10 & d \\
\hline 76.6 & $504-16-1,60$ & -0.73 & +0.52 & d \\
\hline 76.7 & $504-16-1,70$ & -1.41 & +0.30 & a \\
\hline 76.8 & $504-16-1,80$ & -0.96 & +0.86 & d \\
\hline 76.9 & $504-16-1,90$ & -1.18 & +1.06 & $\mathrm{~b}$ \\
\hline 77.0 & $504-16-1,100$ & -1.16 & +1.15 & b \\
\hline 77.1 & $504-16-1,110$ & -1.15 & +1.32 & d \\
\hline 77.3 & $504-16-1,130$ & -1.22 & +1.55 & $\mathrm{~d}$ \\
\hline 77.4 & $504-16-1,140$ & -1.38 & +1.48 & d \\
\hline 77.5 & $504-16-1,150$ & -1.28 & +1.36 & d \\
\hline 77.6 & $504-16-2,10$ & -1.40 & +1.26 & a \\
\hline 77.7 & $504-16-2,20$ & $\left\{\begin{array}{l}-1.65 \\
-1.45\end{array}\right.$ & $\begin{array}{l}+1.34 \\
+1.69\end{array}$ & $\cdot \begin{array}{l}a \\
b\end{array}$ \\
\hline 77.8 & $504-16-2,30$ & -1.56 & +0.98 & $\mathrm{a}$ \\
\hline 77.9 & $504-16-2,40$ & -1.11 & +1.41 & d \\
\hline 78.0 & $504-16-2,50$ & -1.62 & +0.87 & a \\
\hline 78.1 & $504-16-2,60$ & -1.70 & +1.01 & a \\
\hline 78.2 & $504-16-2,70$ & -1.33 & +1.20 & a \\
\hline 78.3 & $504-16-2,80$ & -1.56 & +0.83 & a \\
\hline 78.4 & $504-16-2,90$ & -1.95 & +0.71 & a \\
\hline 78.5 & $504-16-2,100$ & -1.53 & +0.67 & a \\
\hline 78.6 & $504-16-2,110$ & -1.48 & +1.23 & a \\
\hline 79.0 & $504-16-3,0$ & -1.23 & +1.24 & a \\
\hline 79.1 & $504-16-3,10$ & -1.50 & +1.02 & a \\
\hline 79.2 & $504-16-3,20$ & $\left\{\begin{array}{l}-1.70 \\
-1.01\end{array}\right.$ & +1.01 & a \\
\hline 79.3 & $504-16-3,30$ & $\begin{array}{l}-1.01 \\
-0.97\end{array}$ & $\begin{array}{l}+1.64 \\
+1.45\end{array}$ & $\begin{array}{l}\mathrm{b} \\
\mathrm{d}\end{array}$ \\
\hline 79.4 & $504-16-3,40$ & -1.15 & +1.20 & a \\
\hline 79.5 & $504-16-3,50$ & $\left\{\begin{array}{l}-1.59 \\
-0.98\end{array}\right.$ & +1.68 & a \\
\hline 79.6 & $504-16-3,60$ & $\begin{array}{l}-0.90 \\
-1.56\end{array}$ & $\begin{array}{l}+1.05 \\
+1.37\end{array}$ & $\begin{array}{l}\text { D } \\
\text { d }\end{array}$ \\
\hline 79.7 & $504-16-3,70$ & -1.62 & +1.04 & $\mathrm{a}$ \\
\hline 79.8 & $504-16-3,80$ & -1.55 & +1.33 & a \\
\hline 79.9 & $504-16-3,90$ & -1.53 & +1.22 & a \\
\hline 80.9 & $504-17-1,10$ & -1.60 & +0.95 & a \\
\hline 81.6 & $504-17-1,120$ & -1.34 & +1.26 & a \\
\hline 82.4 & $504-17-2,50$ & -1.37 & +1.33 & $\mathrm{a}$ \\
\hline 83.1 & $504-17-2,120$ & -1.08 & +1.33 & a \\
\hline 83.9 & $504-17-3,50$ & -1.33 & +1.10 & a \\
\hline 86.8 & $504-18-2,50$ & -1.80 & +1.62 & d \\
\hline 87.3 & $504-18-2,100$ & -1.27 & +0.71 & a \\
\hline 87.4 & $504-18-2,110$ & -1.16 & +1.39 & b \\
\hline 88.3 & $504-18-3,50$ & -1.66 & +0.80 & $\mathbf{a}$ \\
\hline 88.8 & $504-18-3,100$ & -1.44 & +1.19 & d \\
\hline 89.7 & $504-19-1,50$ & -1.39 & +0.85 & a \\
\hline 91.2 & $504-19-2,50$ & -1.99 & +1.14 & a \\
\hline 91.9 & $504-19-2,120$ & -1.50 & +0.90 & $\mathrm{a}$ \\
\hline 94.1 & $504-20-1,50$ & -1.75 & +0.83 & a \\
\hline 94.8 & $504-20-1,120$ & -1.79 & +0.99 & a \\
\hline 95.7 & $504-20-2,50$ & -1.85 & +1.22 & a \\
\hline 96.2 & $504-20-2,110$ & -1.87 & +1.22 & a \\
\hline 97.1 & $504-20-3,50$ & -1.90 & +1.36 & $\mathrm{a}$ \\
\hline 97.8 & $504-20-3,120$ & -1.51 & +1.49 & a \\
\hline 98.5 & $504-21-1,50$ & -1.66 & +1.70 & a \\
\hline 99.0 & $504-21-1,100$ & -2.03 & +1.81 & a \\
\hline 100.0 & $504-21-2,50$ & -1.45 & +1.23 & $\mathrm{~d}$ \\
\hline 100.5 & $504-21-2,100$ & $\left\{\begin{array}{l}-1.79 \\
-139\end{array}\right.$ & +1.20 & $\mathrm{a}$ \\
\hline & & -1.39 & +1.57 & b \\
\hline 101.5 & $504-21-3,50$ & $\left\{\begin{array}{l}-1.70 \\
-1.72\end{array}\right.$ & +1.68 & a \\
\hline 102.0 & $504-21-3,100$ & -1.62 & $\begin{array}{l}+1.02 \\
+0.93\end{array}$ & $\begin{array}{l}0 \\
a\end{array}$ \\
\hline 102.9 & $504-22-1,50$ & -1.67 & +0.87 & a \\
\hline 103.4 & $504-22-1,100$ & -1.83 & +1.73 & a \\
\hline 104.4 & $504-22-2,50$ & -1.50 & +1.77 & b \\
\hline 104.9 & $504-22-2,100$ & -1.82 & +1.85 & b \\
\hline 107.3 & $504-23-1,50$ & -1.53 & +1.82 & b \\
\hline
\end{tabular}

Table 1. (Continued).

\begin{tabular}{|c|c|c|c|c|}
\hline $\begin{array}{l}\text { Sub-bottom } \\
\text { Depth } \\
\text { (m) }\end{array}$ & $\begin{array}{c}\text { Sample } \\
\text { (level } \\
\text { in } \mathrm{cm} \text { ) }\end{array}$ & $\delta^{18} \mathrm{O}$ & $\delta^{13} \mathrm{O}$ & $\begin{array}{l}\text { Species } \\
\text { Used (see } \\
\text { fns. a-e) }\end{array}$ \\
\hline 107.8 & $504-23-1,100$ & -1.51 & +2.11 & b \\
\hline 111.7 & $504-24-1,50$ & -2.05 & +1.52 & a \\
\hline 112.2 & $504-24-1,100$ & -1.90 & +1.70 & a \\
\hline 113.2 & $504-24-2,50$ & -1.80 & +1.24 & a \\
\hline 116.1 & $504-25-1,50$ & -1.51 & +1.77 & a \\
\hline 116.6 & $504-25-1,100$ & -1.74 & +1.66 & a \\
\hline 118.1 & $504-25-2,100$ & -1.63 & +1.79 & a \\
\hline 119.1 & $504-25-3,50$ & -1.36 & +1.54 & a \\
\hline 120.5 & $504-26-1,50$ & -2.05 & +1.77 & a \\
\hline 121.0 & $504-26-1,100$ & -1.61 & +2.25 & b \\
\hline 122.0 & $504-26-2,50$ & -1.32 & +1.86 & b \\
\hline 123.5 & $504-26-3,50$ & -1.40 & +1.47 & a \\
\hline 124.9 & $504-27-1,50$ & -1.57 & +1.42 & b \\
\hline 125.4 & $504-27-1,100$ & -1.97 & +1.75 & a \\
\hline 126.4 & $504-27-2,50$ & -2.10 & +1.55 & a \\
\hline 126.9 & $504-27-2,100$ & -2.05 & +1.44 & a \\
\hline 127.9 & $504-27-3,50$ & -1.63 & +2.19 & d \\
\hline 129.3 & $504-28-1,50$ & -1.82 & +1.62 & d \\
\hline 130.0 & $504-28-1,120$ & -2.04 & +1.85 & a \\
\hline 130.8 & $504-28-2,50$ & -1.85 & +1.41 & a \\
\hline 133.7 & $504-29-1,50$ & $\left\{\begin{array}{l}-1.84 \\
-1.69\end{array}\right.$ & $\begin{array}{l}+1.40 \\
+1.99\end{array}$ & $\begin{array}{l}\mathrm{a} \\
\mathrm{b}\end{array}$ \\
\hline 134.4 & $504-29-1,120$ & -1.98 & +1.62 & d \\
\hline 135.5 & $504-29-2,120$ & -1.91 & +2.10 & d \\
\hline 136.7 & $504-29-3,50$ & -1.67 & +1.61 & d \\
\hline 137.4 & $504-29-3,120$ & -1.86 & +1.55 & a \\
\hline 138.1 & $504-30-1,50$ & -1.76 & +1.61 & d \\
\hline 138.8 & $504-30-1,120$ & -2.05 & +1.63 & d \\
\hline 139.6 & $504-30-2,50$ & -1.65 & +2.02 & d \\
\hline 140.2 & $504-30-2,112$ & -1.88 & +1.38 & d \\
\hline 141.1 & $504-30-3,50$ & -1.66 & +1.96 & b \\
\hline 142.5 & $504-31-1,50$ & -1.81 & +1.41 & a \\
\hline 143.2 & $504-31-1,120$ & -2.16 & +1.57 & a \\
\hline 144.0 & $504-31-2,50$ & -1.73 & +2.00 & d \\
\hline 144.7 & $504-31-2,120$ & -1.89 & +1.67 & d \\
\hline 146.9 & $504-32-1,50$ & -1.55 & +1.83 & d \\
\hline 147.6 & $504-32-1,120$ & -2.01 & +1.63 & a \\
\hline 148.4 & $504-32-2,50$ & -1.93 & +1.69 & a \\
\hline 149.0 & $504-32-2,113$ & -1.84 & +1.67 & a \\
\hline 149.9 & $504-32-3,50$ & -1.75 & +1.66 & d \\
\hline 151.3 & $504-33-1,50$ & -2.24 & +1.86 & d \\
\hline 156.4 & $504-34-1,120$ & -2.18 & +1.77 & d \\
\hline 157.2 & $504-34-2,50$ & -1.87 & +1.77 & b \\
\hline 157.8 & $504-34-2,113$ & -1.73 & +1.65 & b \\
\hline 160.1 & $504-35-1,50$ & $\left\{\begin{array}{l}-2.30 \\
-1.95\end{array}\right.$ & $\begin{array}{l}+1.99 \\
+1.91\end{array}$ & $\begin{array}{l}\mathrm{a} \\
\mathrm{b}\end{array}$ \\
\hline 160.8 & $504-35-1,120$ & -1.60 & +2.05 & b \\
\hline 161.6 & $504-35-2,50$ & -1.90 & +1.85 & b \\
\hline 162.3 & $504-35-2,120$ & -1.98 & +2.02 & b \\
\hline 163.1 & $504-35-3,50$ & -2.30 & +2.19 & d \\
\hline 164.5 & $504-36-1,50$ & -1.58 & +2.00 & b \\
\hline 165.2 & $504-36-1,120$ & -1.99 & +1.73 & b \\
\hline 166.7 & $504-36-2,120$ & -2.11 & +2.23 & b \\
\hline 169.1 & $504-37-1,50$ & -1.85 & +1.66 & b \\
\hline 169.8 & $504-37-1,120$ & -1.81 & +1.97 & b \\
\hline 171.3 & $504-37-2,120$ & -1.96 & +2.01 & b \\
\hline 175.5 & $504-38-2,120$ & -1.99 & +2.29 & d \\
\hline 176.3 & $504-38-3,50$ & -1.88 & +2.01 & d \\
\hline 182.1 & $504-40-1,50$ & -1.72 & +2.34 & b \\
\hline 182.8 & $504-40-1,120$ & -2.07 & +2.18 & b \\
\hline 183.6 & $504-40-2,50$ & $\left\{\begin{array}{l}-1.96 \\
-1.84\end{array}\right.$ & +1.86 & a \\
\hline 194.5 & $504-43-1,50$ & $\begin{array}{l}-1.04 \\
-1.68\end{array}$ & $\begin{array}{l}+2.60 \\
+1.83\end{array}$ & $\begin{array}{l}\mathrm{b} \\
\mathrm{e}\end{array}$ \\
\hline 197.5 & $504-43-3,50$ & -1.14 & +1.69 & $\mathrm{e}$ \\
\hline 198.2 & $504-43-3,120$ & -1.88 & +1.98 & b \\
\hline 198.5 & $504-44-1,50$ & -1.93 & +2.28 & b \\
\hline 200.0 & $504-44-2,50$ & -2.14 & +2.22 & b \\
\hline 207.2 & $504-44-1,120$ & -1.97 & +1.80 & d \\
\hline
\end{tabular}


Table 1. (Continued).

\begin{tabular}{ccccc}
\hline $\begin{array}{c}\text { Sub-bottom } \\
\text { Depth } \\
(\mathrm{m})\end{array}$ & $\begin{array}{c}\text { Sample } \\
\text { (level } \\
\text { in cm) }\end{array}$ & $\delta^{18} \mathrm{O}$ & $\delta^{13} \mathrm{O}$ & $\begin{array}{c}\text { Species } \\
\text { Used (see } \\
\text { fns. a-e) }\end{array}$ \\
\hline 219.6 & $504-49-2,16$ & -1.87 & +2.23 & $\mathrm{~b}$ \\
222.5 & $504-50-1,50$ & -1.87 & +2.28 & $\mathrm{~d}$ \\
230.5 & $504-52-1,50$ & -1.93 & +2.51 & $\mathrm{~b}$ \\
231.0 & $504-52-1,100$ & -2.23 & +2.42 & $\mathrm{~b}$ \\
234.5 & $504-53-1,50$ & -1.69 & +1.59 & $\mathrm{~b}$ \\
\hline
\end{tabular}

${ }^{a} G$. ruber (both quantities).

${ }^{b} G$. sacculifer (both quantities).

${ }^{\mathrm{c}} N$. dutertrei (both quantities).

d Mixed G. ruber and G. sacculifer.

e G. sacculifer and Globoquadrina altispira.

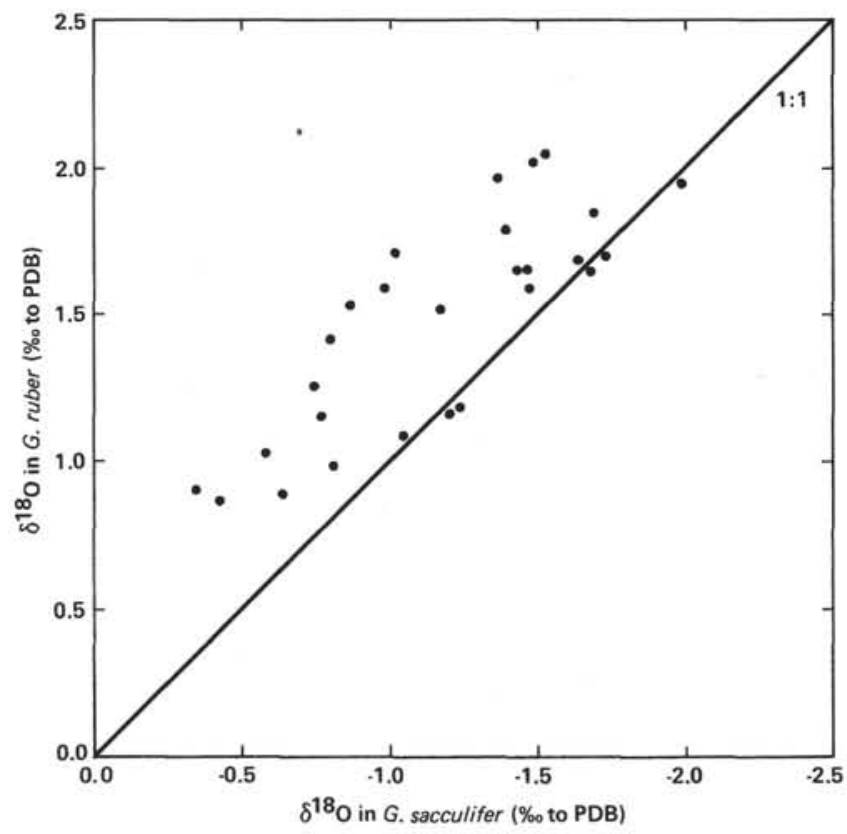

Figure 1. Relation between $\delta^{18} \mathrm{O}$ values in $G$. ruber and in $G$. sacculifer for those samples in which both species were analyzed.

data for more than one species form a single curve, this procedure obscures some of the information and may in some cases be seriously misleading. In fact we have not used the data for $N$. dutertrei in constructing Figure 2 , and have plotted the ${ }^{18} \mathrm{O}$ values for $G$. sacculifer after subtracting 0.35 per mil from the measured values (which would bring the data in Figure 1 closer to a 1:1 fit). The ${ }^{13} \mathrm{C}$ values in Figure 2 represent values for $G$. ruber only.

Figure 2 depicts (subject to the reservation expressed above) the oxygen and carbon isotope record for the past 1.8 m.y. Figure 3 shows an age-depth plot for the site. Figure 3 was constructed in two steps. First, the biostratigraphic data were plotted, giving a rough (but consistent) line. This line was used to identify the isotope stages defined by Emiliani (1955) and by Shackleton and Opdyke (1973) (several of the stages have characteristics that enable them to recognized visually). The ages of these stage boundaries were taken from the lat- ter publication, modified to take account of the impact on the paleomagnetic scale of more recent decay constants for $\mathrm{K}-\mathrm{Ar}$ dating, and placed on the age-depth plot. The ages assigned to the biostratigraphic datums in Table 2 are also adjusted for the newly adopted decay constants.

Overall, the accumulation rate is surprisingly uniform. In Figure 4 the record obtained with the hydraulic piston corer is compared with a record obtained with a conventional piston corer (Core V28-238 [Shackleton and Opdyke, 1973]). The most striking difference between the records is the range of variation, which is considerably greater in the new data. This is as would be expected from the higher sediment accumulation rate and is supported by the analysis of benthic foraminifers for the same region (Ninkovich and Shackleton, 1975), which shows a yet larger variation. The demonstration of a high degree of variation with planktonic foraminifers is important, however, because the previously published records have been based on benthic foraminifers. This has led some workers to feel that the planktonic foraminifers in the Pacific display an anomalously low isotopic variation for which new interpretations must be sought (Erez, 1979), despite the fact that Peng et al. (1977) showed that the variation is entirely consistent with variations in sediment accumulation rate and the effects of bioturbation.

\section{DISCUSSION OF RESULTS}

\section{Long-Term Variability in the Oxygen Isotope Record}

Shackleton and Opdyke (1973) drew attention to the fact that in the record they examined the isotopic extremes (lows during the glacial periods, and peaks during the interglacial periods) remained rather stable through the past million years. Shackleton (1977a) went further and suggested that deviations from this tendency were a characteristic of cores that were particularly badly affected by the interplay of dissolution and bioturbation in a low accumulation regime. However, we see in the Hole 504 record that there are significant differences between both one glacial extreme and another and one interglacial extreme and another. For example, Stages 16 and 22 appear much more intensely "glacial" than most of the even-numbered stages, whereas Stage 11 appears extremely "interglacial". Unfortunately, no detailed benthic isotope records extending to Stage 16 exist, so we do not know whether this extreme represents a low in ocean isotopic composition (i.e., a period of extremely extensive continental glaciation) or merely a period of substantial cooling of surface waters in the region. Insofar as Stage 11 is concerned, data from Core V19-28 (Ninkovich and Shackleton, 1975) do not suggest that significantly less ice existed at that time, and indeed such a large excursion, if explained in terms of ocean isotopic composition, would necessitate the melting of the entire Antarctic ice sheet. However, several previous workers have identified an extremely warm interglacial immediately after the extinction of the radiolarian Stylatractus universus (Hays, 1965; Sachs, 1973), and we now know 

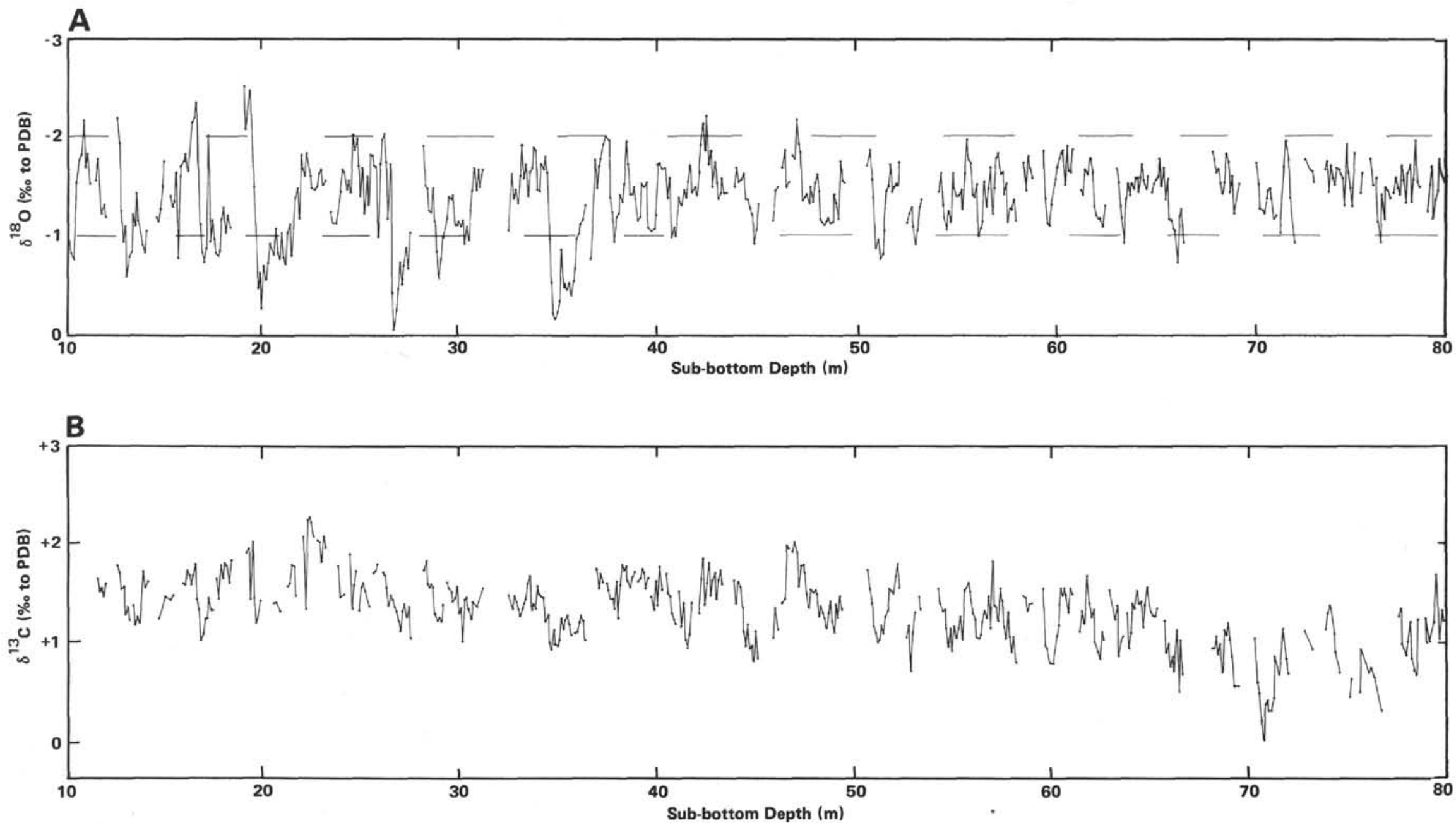

Figure 2. A. Oxygen isotope record for the Pleistocene section of Hole 504. Analyses based on G. ruber and G. sacculifer, the latter adjusted by -0.35 per mil. B. Carbon isotope record for the Pleistocene section of Hole 504. Analysis based on $G$. ruber only. 


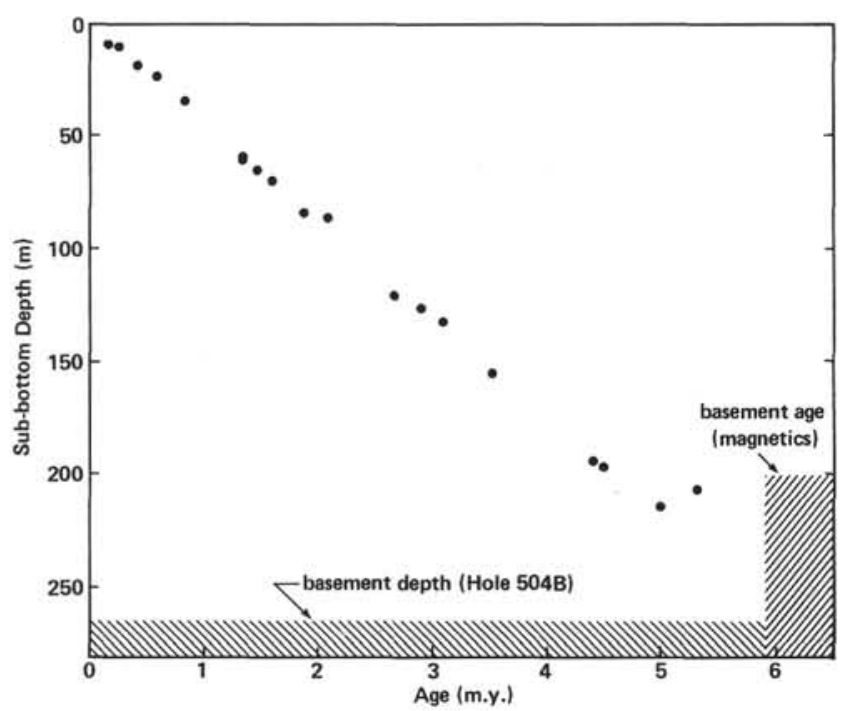

Figure 3. Age-depth plot for Hole 504, using stage boundaries and biostratigraphic datums listed in Table 2 .

that this extinction occurred at the Stage 11/12 boundary (Hays and Shackleton, 1976).

\section{High-Frequency Variation in the Oxygen Isotope Record}

Even more important than the record's long-term trends are its high-frequency variations. There are many points in the record where within a relatively short interval (say 20,000 years) the isotopic value changes from one extreme to the other and back. That is to say, at the level of spectral analysis of which the human eye is capable, there is considerable power in the range that may be related to precession in the earth's orbital system.

Preliminary spectral analysis in collaboration with J. Imbrie and N. Pisias confirms the presence of spectral energy at a period near 23,000 years, although unfortunately spectral analysis is rendered uncertain by the frequent gaps between measurements in successive cores. From the geological point of view it is very important to know that during the lower Pleistocene, in particular, such a large degree of short-period variability prevailed.

\section{The Carbon Isotope Record}

Very few carbon isotope records have been published with which the present data may be compared. Shackleton and Cita (1979) obtained a long carbon isotope record for benthic foraminifers from Hole 397. However, it is difficult to make a useful comparison between the two records other than to comment that both show a trend to isotopically lighter ${ }^{13} \mathrm{C}$ values near the base of the Pleistocene. Since Hole 397 was sampled before the advent of the hydraulic piston corer, core disturbance prevents exact correlation with Hole 504.

\section{The Pliocene Record}

Figure 5 shows the preliminary oxygen and carbon isotope data so far obtained for the Pliocene section of Hole 504 , which was sampled at intervals of about 1.5 meters (equivalent to about 20,000 years). This record supports the conclusion reached by Shackleton and Opdyke (1977) on the basis of an oxygen isotope record derived from benthic foraminifers that the onset of major glacial/interglacial fluctuations occurred about the end of the Gauss magnetic epoch. However, more detailed work will be necessary to determine whether diagenetic alteration has affected the values in the lower part of the section. This problem was encountered by Keigwen (1979), who analyzed material from Hole 157 on similarly aged crust south of the Panama Basin. The carbon isotope record is broadly similar to that of Hole 397 (which is in the North Atlantic near Cape Bojador, Morocco), in that they both display less high frequency variability

Table 2. Datums used for age-depth plot in Figure 3.

\begin{tabular}{|c|c|c|c|c|}
\hline Datum & Sample & $\begin{array}{l}\text { Sub-bottom } \\
\text { Depth (m) }\end{array}$ & Age (m.y.) & Source \\
\hline Stage $6 / 7$ boundary & $504-1-1,35 \mathrm{~cm}$ & 10.35 & 0.188 & Morley and Hays (1981) \\
\hline Stage $7 / 8$ boundary & $504-1-2,125 \mathrm{~cm}$ & 12.75 & 0.244 & Morley and Hays (1981) \\
\hline Stage $11 / 12$ boundary & $504-3-1,80 \mathrm{~cm}$ & 19.60 & 0.421 & Morley and Hays (1981) \\
\hline Stage $15 / 16$ boundary & $504-4-3,15 \mathrm{~cm}$ & 26.35 & 0.579 & Morley and Hays (1981) \\
\hline Stage $22 / 23$ bondary & $504-6-3,100 \mathrm{~cm}$ & 36.00 & 0.828 & Berggren et al. (1980) \\
\hline Helicosphaera sellii $\mathrm{LAD}^{\mathrm{a}}$ & $504-12-2,90 \mathrm{~cm}$ & 60.80 & 1.37 & J. Backman, pers. comm. (1981) \\
\hline $\begin{array}{l}\text { Cyclococcolithus macintyrei } \\
\text { LAD }\end{array}$ & $504-13-1,150 \mathrm{~cm}$ & 66.30 & 1.46 & $\begin{array}{l}\text { J. Backman, pers. comm., (1981) Backman } \\
\text { (1981) }\end{array}$ \\
\hline Globoquadrina fistulosus LAD & $504-14-3,50 \mathrm{~cm}$ & 70.90 & 1.60 & This chapter, Berggren, et al. (1980) \\
\hline Discoaster brouweri LAD & $504-17-3,90 \mathrm{~cm}$ & 84.20 & 1.88 & $\begin{array}{l}\text { J. Backman, pers. comm. (1981), Backman } \\
\text { (1981) }\end{array}$ \\
\hline G. exilis LAD & $504-18-3,50 \mathrm{~cm}$ & 88.30 & 2.1 & This chapter \\
\hline D. tamalis LAD & $504-26-2,17 \mathrm{~cm}$ & 121.67 & 2.67 & J. Backman, pers. comm. (1981) \\
\hline G. fistulosus $\mathrm{FAD}$ & $504-27-2,100 \mathrm{~cm}$ & 126.90 & 2.9 & $\begin{array}{l}\text { This chapter, Thompson and Sciarrillo } \\
\text { (1979) } \\
\text { Thunell (1981) }\end{array}$ \\
\hline G. altispira $\mathrm{LAD}$ & $504-27-3,50 \mathrm{~cm}$ & 127.90 & 2.9 & $\begin{array}{l}\text { This chapter, Thompson and Sciarrillo } \\
\text { (1979), Thunell (1981) }\end{array}$ \\
\hline S. seminulina LAD & $504-29-1,50 \mathrm{~cm}$ & 133.70 & 3.1 & $\begin{array}{l}\text { This chapter, Thompson and Sciarrillo } \\
\text { (1979), Thunell (1981) }\end{array}$ \\
\hline $\begin{array}{l}\text { Reticulofenestra pseudoumbilica } \\
\text { LAD }\end{array}$ & $504-34-1,120 \mathrm{~cm}$ & 156.40 & 3.4 & J. Backman, pers, comm. (1981) \\
\hline Nitzschia cylindrica LAD & $504-43-1,14 \mathrm{~cm}$ & 194.14 & 4.41 & Sancetta (this vol.) \\
\hline$N$. jouseae $\mathrm{FAD}^{6}$ & $504-43-3,14 \mathrm{~cm}$ & 197.14 & 4.49 & Sancetta (this vol.) \\
\hline G. dehiscens LAD & $504-46-1,50 \mathrm{~cm}$ & 206.50 & 5.3 & $\begin{array}{l}\text { This chapter, Saito et al. (1975), Thunell } \\
\text { (1981) }\end{array}$ \\
\hline T. miocenica L.AD & $504-48, \mathrm{CC}$ & 215.30 & 5.0 & Sancetta (this vol.) \\
\hline
\end{tabular}




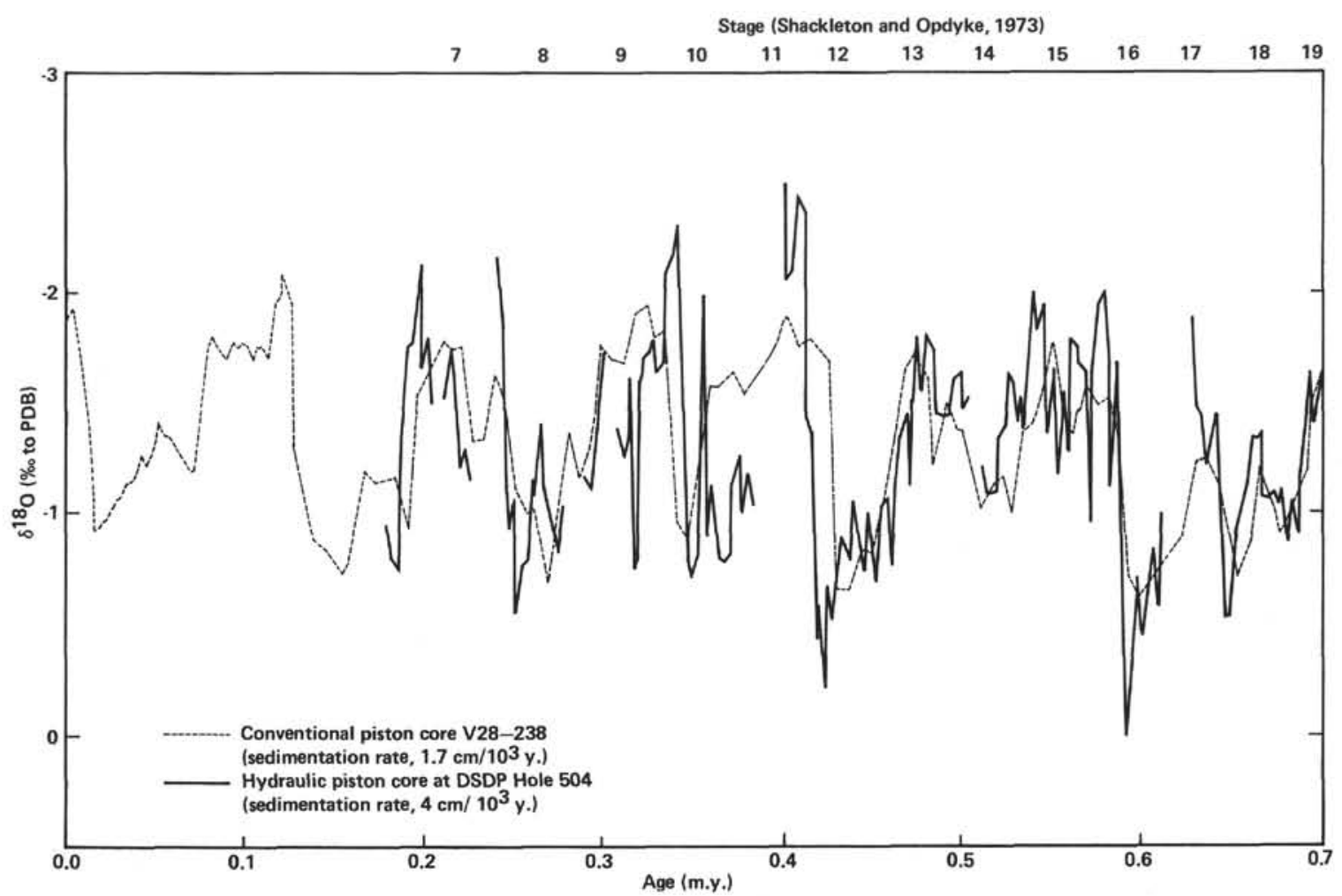

Figure 4. Oxygen isotope record derived from Hole 504 for the past 700,000 years, compared with record derived from conventional piston core V28-238 (Shackleton and Opdyke, 1973), both plotted to a uniform accumulation rate. Approximate position if isotope stages indicated at top.

about a mean that is isotopically a little lighter than the light extremes in the upper part of the record.

\section{CONCLUSIONS}

The sediments of Hole 504 are the most important materials thus far obtained for the study of climatic variability during the lower Pleistocene and late Pliocene. Even with only a single penetration of the site with the hydraulic piston corer, which leaves a stratigraphic gap of several tens of centimeters in every 4.5 meter section, the record is extremely valuable. If the opportunity to resample the site arose, a major research effort would be warranted to investigate the onset of glaciation and the factors that contribute to the progressive change in glacial intensity during the Pleistocene.

\section{ACKNOWLEDGMENTS}

We are very grateful to the members of the Leg 69 Shipboard Scientific Party for their care in recovering the sedimentary section despite the fact that their real interest lay in the basalts beneath; even more, we thank them for their generosity in taking so many samples onboard ship. We warmly thank C. Solanki for his laboratory assistance, Jan Backman for his careful determination of nannofossil datums, and Connie Sancetta, who reviewed the manuscript with considerable care. Work in Cambridge was supported by NERC grant GR3/1762.

\section{REFERENCES}

Backman, J. E., 1981. Quantitative biochronology of nannofossils at the Plio-Pleistocene boundary. Terra Cognita (special issue), p. 115. (Abstract)

Berggren, W. A., Burckle, L. H., Cita, M. B., Cooke, H. B. S., Funnell, B. M., Gartner, S., Hays, J. D., Kennett, J. P., Opdyke, N.
D., Pastouret, L., Shackleton, N. J., and Takayanagi, Y., 1980. Towards a Quaternary time scale. Quat. Res., 13:277-302.

Emiliani, C., 1955. Pleistocene temperatures. J. Geol. 63:538-578.

Erez, J., 1979. Modification of the oxygen-isotope record in deep-sea cores by Pleistocene dissolution cycles. Nature, 281:535-538.

Hays, J. D., 1965. Radiolaria and late Tertiary and Quaternary history of Antarctic seas. Antarct. Res. Ser., 5:125-184.

Hays, J. D., and Shackleton, N. J., 1976. Globally synchronous extinction of the radiolarian Stylatractus universus. Geology, 4: 649-652.

Keigwen, L. D., Jr., 1979. Late Cenozoic stable isotope stratigraphy and paleoceanography of DSDP sites from the east equatorial and central north Pacific ocean. Earth Planet. Sci. Lett., 45: 361-382.

Morley, J. J., and Hays, J. D., 1981. Towards a high-resolution, global, deep-sea chronology for the last 750,000 years. Earth Planet. Sci. Lett., 53:279-295.

Ninkovich, D., and Shackleton, N. J., 1975. Distribution, stratigraphic position and age of ash layer "L", in the Panama Basin region. Earth Planet. Sci. Lett., 27:20-34.

Peng, T.-H., Broecker, W. S., Kipphut, G., and Shackleton, N. J., 1977. The relation of sediment mixing to the distortion of climatic records in the deep sea sediments. In Andersen, N. R., and Malahoff, A. (Eds.), The Fate of Fossil Fuel $\mathrm{CO}_{2}$ in the Oceans, pp. 355-373.

Sachs, H. M., 1973. North Pacific radiolarian assemblages and their relationship to oceanographic parameters. Quat. Res., 3:73-88.

Saito, T., Burckle, L. H., and Hays, J. D., 1975. Late Miocene to Pleistocene biostratigraphy of equatorial Pacific sediments. In Saito, T., and Burckle, L. H., (Eds.), Late Neogene Epoch Boundaries: New York (Micropaleontology Press), pp. 226-244.

Shackleton, N. J., 1977a. Oxygen isotope stratigraphy of the Middle Pleistocene. In Shotton, F. W. (Ed.), British Quaternary Studies: Oxford (Clarendon Press), pp. 1-16.

, 1977b. The oxygen isotope stratigraphic record of the Late Pleistocene. In Mitchell, G. F., and West, R. G. (Eds.), The Changing Environmental Conditions in Great Britain and Ireland 
During the Devensian (Last) Cold Stage: Philos. Trans. R. Soc. London, Ser. B, 280:169-179.

Shackleton, N. J., and Cita, M. B., 1979. Oxygen and carbon isotope stratigraphy of benthic foraminifers at Site 397: detailed history of climatic change during the late Neogene. In von Rad, U., Ryan, W. B. F., et al., Init. Repts. DSDP, 47, Pt. 1: Washington (U.S. Govt. Printing Office), 433-445.

Shackleton, N. J., and Opdyke, N. D., 1973. Oxygen isotope and palaeomagnetic stratigraphy of equatorial Pacific core V28-238: oxygen isotope temperatures and ice volumes on a $10^{5}$ year and $10^{6}$ year scale. Quat. Res., 3:39-55.

, 1977. Oxygen isotope and palaeomagnetic evidence for early Northern Hemisphere glaciation. Nature, 270:216-219.

Thompson, P. R., and Sciarrillo, J. R., 1978. Planktonic foraminiferal biostratigraphy in the equatorial Pacific. Nature, 276:29-33.

Thunell, R. C., 1981. Late Miocene-early Pliocene planktonic foraminiferal biostratigraphy and paleoceanography of low-latitude marine sequences. Mar. Micropalaeontol., 6:71-90.

\section{APPENDIX}

\section{Biostratigraphy}

For the purpose of producing the age-depth plot in Figure 3, several biostratigraphic datums were located in the samples available. For the nannofossils, these were determined by Dr. Jan Backman; for the foraminifers, by N. J. Shackleton. The positions of the foraminiferal datums are estimated on the basis of rather small samples, and may be subject to some modification. Present estimates of the position of each useful datum, its assigned age, and the source of this estimate are given in Table 2 . This table contains only calcareous datums for the past 4 m.y.; in general, the agreement with the siliceous datums determined by Sancetta (this volume) is good. The three siliceous datums from Sancetta (this volume) in the lower part of the section suggest that the age of $5.3 \mathrm{~m} . \mathrm{y}$. assigned to the extinction of Globoquadrina dehiscens, which is based on Saito et al. (1975) as modified by Thunell (1981), is probably too great by about $0.3 \mathrm{~m} . \mathrm{y}$.
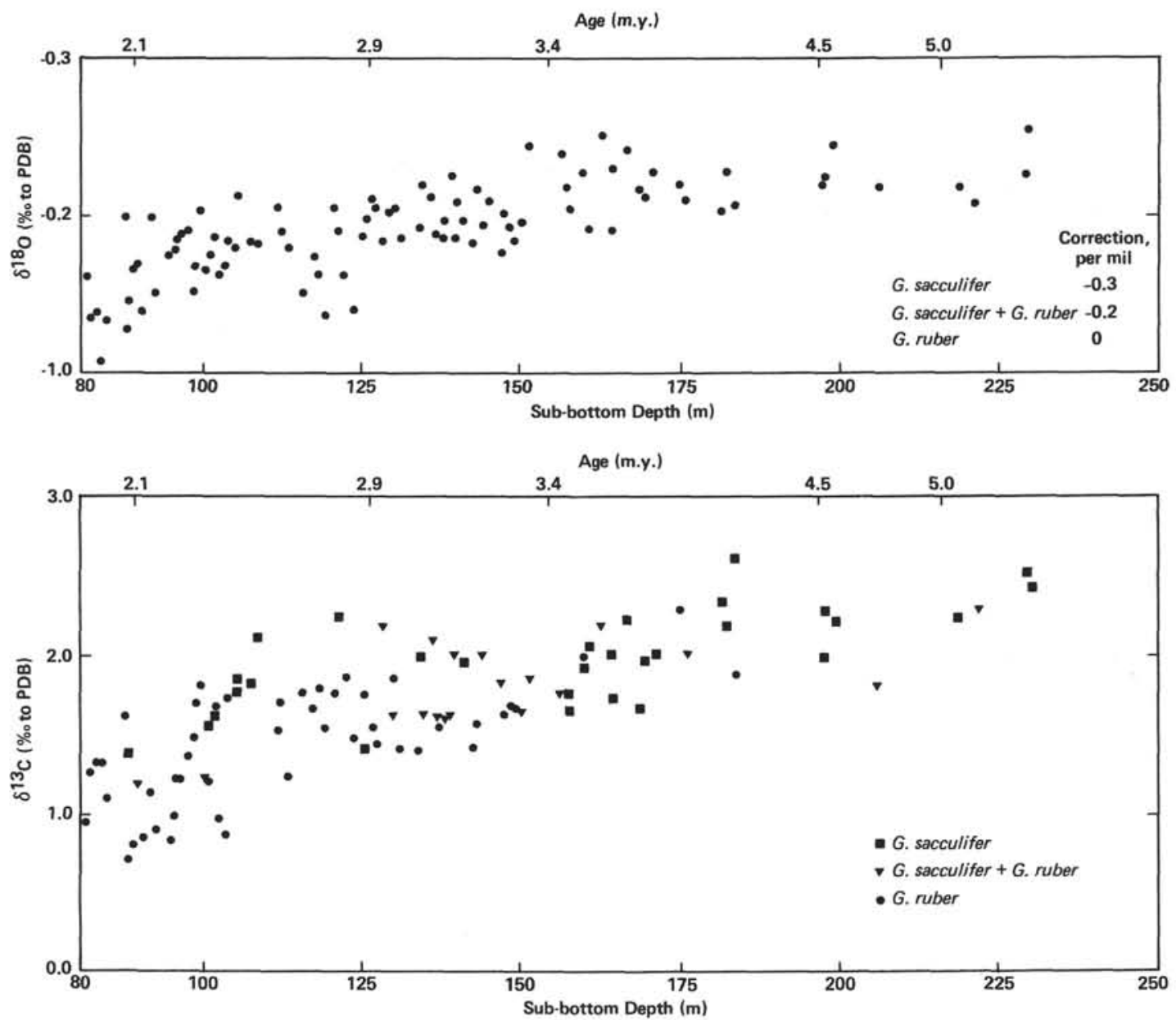

Figure 5. Preliminary ${ }^{18} \mathrm{O}$ and ${ }^{13} \mathrm{C}$ data for the Pliocene section of Hole $504 .{ }^{18} \mathrm{O}$ values for $G$. sacculifer are plotted after subtracting 0.35 per mil to make them comparable to the data for $G$. ruber. Where a mixture of the two species was analyzed, a correction of 0.2 per mil was used. The ${ }^{13} \mathrm{C}$ values for $G$. sacculifer are not adjusted but can be distinguished by symbol shape. 